\title{
NON-LINEAR HYBRID-MODE RESONANT FORCED OSCILLATIONS OF SAGGED INCLINED CABLES AT AVOIDANCES
}

\author{
Giuseppe Rega \& Narakorn Srinil \\ Department of Structural and Geotechnical Engineering, University of Rome 'La Sapienza', \\ via A. Gramsci 53, Rome 00197, Italy
}

\begin{abstract}
We investigate non-linear forced oscillations of sagged inclined cables under planar 1:1 internal resonance at avoidance. To account for frequency avoidance phenomena and associated hybrid modes actually distinguishing inclined cables from horizontal cables, asymmetric inclined static configurations are considered. Emphasis is placed on highlighting nearly tuned 1:1 resonant interactions involving coupled hybrid modes. The inclined cable is subjected to a uniformlydistributed vertical harmonic excitation at primary resonance of a high-frequency mode. Approximate non-linear partial-differential equations of motion, capturing overall displacement coupling and dynamic extensibility effect, are analytically solved based on a multi-mode discretization and a second-order multiple scales approach. Bifurcation analyses of both equilibrium and dynamic solutions are carried out via a continuation technique, highlighting the influence of system parameters on internally resonant forced dynamics of avoidance cables. Direct numerical integrations of modulation equations are also performed to validate the continuation prediction and characterize non-linear coupled dynamics in post-bifurcation states. Depending on the elasto-geometric (cable sag and inclination) and control parameters, and on assigned initial conditions, the hybrid modal interactions undergo several kinds of bifurcations and non-linear phenomena, along with meaningful transition from periodic to quasi-periodic and chaotic responses. Moreover, corresponding spatio-temporal distributions of cable non-linear dynamic displacement and tension are manifested.
\end{abstract}

Keywords: inclined cable, frequency avoidance, hybrid mode, forced vibration, internal resonance, bifurcation analysis, chaos 


\section{INTRODUCTION}

Arbitrarily inclined and sagged cables are found in many engineering applications, often also integrated into large structures for specific purposes. Offshore marine cables, cable-stayed bridges, commuter cable-car networks, cable ski lifts and overhead transmission conductors are a few examples. Unfortunately, highly flexible cables exhibit inherently low structural damping; therefore external disturbances may easily cause a number of unexpected non-linear dynamic phenomena entailing cable vibration-induced problems. Therefore, dynamics engineers are often confronted with difficulties pertinent to such time-dependent circumstances.

Generally speaking, the great majority of research literature on non-linear vibrations of suspended cables deals with parabolic horizontal [1] or nearly taut - typically symmetric inclined cables [2-6]. In numerous applications, however, this is not the case as cables can be suspended over great distances, thereby experiencing significant sags as well as different support levels. Hence, sagged asymmetric inclined cables are encountered. As a matter of fact, little attention has been paid to both the theoretical modeling and dynamic behavior of inclined cables. Large-amplitude planar/non-planar free vibrations of horizontal/inclined cables have been numerically studied by Takahashi and Konishi [7] and Srinil et al. [8, 9], with 1:1 or 2:1 internal resonance effects being highlighted in the latter. Recently, based on a general kinematic condensation-free modeling, non-linear finite-amplitude free dynamics of horizontal/inclined cables have been analytically characterized and compared [10, 11], focusing on planar 2:1 resonances existing away from frequency crossover [12] and avoidance [13-15] regions in the associated frequency spectra.

The main objective of this paper is to investigate the effect of system asymmetry due to sagged inclined configurations on non-linear planar forced oscillations of inclined cables. Particular attention is placed on hybrid modal interactions due to a nearly-tuned 1:1 internal resonance of avoided-crossing (veering) frequencies. From both a theoretical and practical viewpoint, frequency avoidance phenomena - together with the coexisting hybrid modes - are 
actually concerned with planar dynamics, distinguishing inclined cables from horizontal cables that exhibit crossover phenomena of symmetric/anti-symmetric frequencies. Experimental investigations have also evidenced the occurrence of frequency avoidance [16, 17], hybrid modes and specific features of modal interactions in inclined cables [17]. Within the framework of vibration suppression, $\mathrm{Xu}$ and $\mathrm{Yu}[18]$ have found that frequency avoidance may cause a dramatic reduction in modal damping ratios supplied by external dampers locally attached to inclined stay cables. On the contrary, the mode localization theory - which is associated with frequency avoidance - may be somehow applied to realize a passive control of vibration propagation in structures [19]. This disadvantageous/advantageous relevance is the reason why it is of great practical significance to fully understand the non-linear forced dynamic behavior of avoidance cables, with respect to the well-known crossover cables [1].

The paper is organized as follows. Section 2 presents the approximate planar model of an elastic inclined cable and the associated non-linear equations of motion accounting for overall displacement coupling and dynamic extensibility [10]. The key solution of sagged inclined configurations, based on a cubic space polynomial, is underlined, since it entails the system asymmetry from which linear frequency avoidances and associated hybrid modes are originated. Based on a multi-mode Galerkin discretization and a second-order multiple scales approach, coupled amplitude-dependent solution due to primary external and 1:1 internal resonances is summarized in Sect. 3. Depending on the elasto-geometric parameter, significant contributions of hybrid or asymmetric, also non-resonant, modes associated with quadratic nonlinearities are discussed in Sect. 4 for different avoidance cables. By means of frequency- and force-response diagrams, local bifurcation studies of steady- and dynamic-state solutions are performed in Sect. 5, showing the influence of system parameters on resonant dynamics. Several meaningful nonlinear phenomena are highlighted via direct numerical integrations of modulation equations, and the space-time varying distributions of non-linear dynamic displacement and tension are examined. The paper ends with some concluding remarks. 


\section{GOVERNING EQUATIONS}

In a fixed Cartesian co-ordinate system, Figure 1a displays an elastic simply-supported suspended cable with arbitrary angle $\theta$ of inclination. Keeping the horizontal span $X_{H}$ fixed, the vertical span $Y_{H}$ is varied to attain a desired inclination, and the horizontal component $H$ of cable static tension is adjusted to attain a desired cable sag value $d$. The planar static configuration $y(x)$ is attained under influence of gravity $g$, with $x$ being the space variable, whereas the coupled planar dynamics are described by the horizontal $u$ and vertical $v$ displacements measured from the static equilibrium at point $x$ and time $t$. Here, the cable is driven by a uniformly-distributed vertical harmonic excitation, i.e., $F(x, t)=F \cos \Omega t$, with $F$ and $\Omega$ being the amplitude and frequency of excitation, respectively. In the following, a prime (dot) expresses differentiation with respect to $x(t)$. To facilitate the parametric study, the following dimensionless variables are introduced:

$$
\begin{aligned}
& \tilde{x}=\frac{x}{X_{H}}, \tilde{y}=\frac{y}{X_{H}}, \tilde{d}=\frac{d}{X_{H}}, \tilde{u}=\frac{u}{X_{H}}, \tilde{v}=\frac{v}{X_{H}}, \tilde{t}=\frac{t}{X_{H}} \sqrt{\frac{g H}{w_{C}}}, \\
& \alpha=\frac{E A}{H}, \tilde{c}=c \frac{X_{H}}{H} \sqrt{\frac{g H}{w_{C}}}, \tilde{F}=F \frac{X_{H}}{H}, \tilde{\Omega}=\Omega X_{H} \sqrt{\frac{w_{C}}{g H}},
\end{aligned}
$$

in which $E$ is the Young's Modulus, $A$ the uniform circular cross-sectional area, $w_{C}$ the selfweight per unit unstretched length, $c$ the coefficient of linear viscous damping. Based on the assumptions of small axial deformation and moderately large vibration amplitudes, the extensional space-time dependent axial strain, neglecting the effects of torsional, flexural and shear rigidities, is expressed, through the Lagrangian approximation, as [10]

$$
e_{d} \approx \frac{1}{1+y^{\prime 2}}\left(u^{\prime}+y^{\prime} v^{\prime}+\frac{1}{2}\left(u^{\prime 2}+v^{\prime 2}\right)\right)
$$

Due to the strain energy of cable non-linear stretching, the approximate non-linear partial differential equations describing forced damped planar vibration of inclined cables read [10]

$$
\rho \ddot{u}+c \rho \dot{u}=\left\{u^{\prime}+\frac{\alpha}{\rho^{3}}\left(u^{\prime}+y^{\prime} v^{\prime}\right)+\frac{\alpha}{\rho^{3}}\left(u^{\prime 2}+y^{\prime} u^{\prime} v^{\prime}+\frac{1}{2}\left(u^{\prime 2}+v^{\prime 2}\right)\right)+\frac{\alpha}{2 \rho^{3}}\left(u^{\prime 3}+u^{\prime} v^{\prime 2}\right)\right\}^{\prime},
$$


$\rho \ddot{v}+c \rho \dot{v}=\left\{v^{\prime}+\frac{\alpha}{\rho^{3}}\left(y^{\prime} u^{\prime}+y^{\prime 2} v^{\prime}\right)+\frac{\alpha}{\rho^{3}}\left(u^{\prime} v^{\prime}+y^{\prime} v^{\prime 2}+\frac{y^{\prime}}{2}\left(u^{\prime 2}+v^{\prime 2}\right)\right)+\frac{\alpha}{2 \rho^{3}}\left(u^{\prime 2} v^{\prime}+v^{\prime 3}\right)\right\}^{\prime}+F \rho \cos \Omega t$,

in which $\rho=\sqrt{1+y^{\prime 2}}$. Zero displacements at boundaries are considered and the tilde notation is omitted for brevity. Accounting for overall inertia effect [20], this system - besides revealing geometrically quadratic and cubic nonlinearities due to cable sag and axial stretching - explicitly captures the interaction of $u$ and $v$ dynamic amplitudes.

Solving the relevant static problem with moderate sag assumption [10], the key closed-form solution for static inclined configuration - that is, in non-dimensional form $y=\tan \theta+z-$ is obtained, up to cubic order of $x$, through [12]

$$
Z \approx \frac{1}{2} x(1-x)\left(1+\frac{\varepsilon^{*}}{6}(1-2 x)\right)+O\left(\varepsilon^{*^{2}}\right)
$$

in which $Z=z /\left(w_{C} X_{H} \sec \theta / H\right)$ and $\varepsilon^{*}=w_{C} X_{H} \sin \theta / H$ are dimensionless parameters. It is worth mentioning that, by accounting for the asymmetry to first order of the small parameter $\varepsilon^{*}$, the ensuing inclined profile is no longer parabolic or symmetric. As verified by numerical solution of the exact static equation [10], Eq.(5) is valid even if the values of sag-to-span ratio $d$ are somewhat greater than the limiting value (1:8) of parabolic profile. Thus, with respect to the parabolic configuration commonly considered also in inclined cables literature [2-5], this straightforward solution provides a greater sag range useful in several engineering applications.

Based on assumed sine-based series, the linear eigenvalue problem of free undamped motion of Eqs.(3)-(4) is solved by the Galerkin method, yielding system eigenfrequencies and eigenfunctions of coupled $u$ and $v$ displacements [10] which depend on the elasto-geometric inclined cable parameter $[13,14]$

$$
\lambda^{2}=\left(w_{C} S \cos \theta\right)^{2} E A / T_{a}^{3},
$$

where $S$ is the cable equilibrium length and $T_{a}$ the static tension at the cable point where the local inclination angle is approximately equal to $\theta$. 
In Fig.1b, frequency avoidance phenomena are illustrated by loci of the lowest four planar frequencies plotted versus $\lambda / \pi$ for the inclined cable with $\theta=30^{\circ}$. It is seen that, as $\lambda / \pi$ (as well as cable sag) slowly increases, two loci progressively approach each other at $\lambda / \pi \approx 2,4$ and so on, thereafter abruptly diverging from each other. The coexisting $(r, s)$ modal shapes are essentially hybrid (asymmetric) at avoidances due to a mixture of symmetric/anti-symmetric modes, as exemplified in Fig. 2 for $\theta=30^{\circ}$ and $45^{\circ}$. According to linear theory, dynamic tensions of such hybrid modes are substantially high [14], in contrast with the corresponding crossover cables which exhibit trivial (non-trivial) dynamic tensions of the coexisting anti-symmetric (symmetric) modes [12]. To describe sensitivity to system asymmetry, the nearness to avoidedcrossing frequencies is achieved by varying the non-zero detuning parameter governing the 1:1 resonance (see Sect. 5).

To analytically treat the temporal problem, Eqs. (3) and (4) are cast in state-space (first-order) form [21]. By accounting for the orthonormality properties of linear eigenfunctions, the derived equations are projected onto the infinite-dimensional eigenbasis by letting

$$
U^{J}=\sum_{m=1}^{\infty} f_{m} \zeta_{m}^{J}, V^{J}=\sum_{m=1}^{\infty} p_{m} \zeta_{m}^{J}
$$

in which $J=1,2, U^{1}=u, U^{2}=v, V^{1}=\dot{u}, V^{2}=\dot{v}, \zeta_{m}^{1}=\phi_{m}, \zeta_{m}^{2}=\varphi_{m}, f_{m}$ and $p_{m}$ being the unknown displacement and velocity coordinates associated with both the horizontal $\left(\phi_{m}\right)$ and vertical $\left(\varphi_{m}\right)$ shape functions of the $m$ mode. The Galerkin method is then applied, thereby leading to the infinite set of non-linearly coupled ordinary differential equations:

$$
\begin{aligned}
& \dot{f}_{m}-p_{m}=0, \\
& \dot{p}_{m}+2 \mu_{m} p_{m}+\omega_{m}^{2} f_{m}=\sum_{i=1}^{\infty} \sum_{j=1}^{\infty} \Lambda_{m i j} f_{i} f_{j}+\sum_{i=1}^{\infty} \sum_{j=1}^{\infty} \sum_{k=1}^{\infty} \Gamma_{m i j k} f_{i} f_{j} f_{k}+Z_{m} \cos \Omega t,
\end{aligned}
$$

for $m=1,2, \ldots, \infty$, wherein $\omega_{m}$ is the $m$ natural frequency. The modal damping and external forcing terms read $2 \mu_{m}=c \int_{0}^{1} \rho\left(\phi_{m}^{2}+\varphi_{m}^{2}\right) d x, Z_{m}=F \Upsilon_{m}=F \int_{0}^{1} \rho \varphi_{m} d x$, respectively. 
Note that, for crossover cables, the associated $Z_{r}\left(Z_{s}\right)$ is always trivial (non-trivial) corresponding to the coexisting $r$ th anti-symmetric (sth symmetric) mode, whereas for inclined cables at avoidances $Z_{r}$ and $Z_{s}$ are never trivial owing to the relevant hybrid (asymmetric) modes. The quadratic and cubic non-linear coefficients governing $(m, i, j, k)$ overall displacement coupling are given, respectively, by [10]

$$
\begin{aligned}
& \Lambda_{m i j}=-\alpha \int_{0}^{1} \frac{1}{\rho^{3}}\left\{\phi_{m}^{\prime}\left(\frac{3}{2} \phi_{i}^{\prime} \phi_{j}^{\prime}+y^{\prime} \phi_{i}^{\prime} \varphi_{j}^{\prime}+\frac{1}{2} \varphi_{i}^{\prime} \varphi_{j}^{\prime}\right)+\varphi_{m}^{\prime}\left(\frac{y^{\prime}}{2} \phi_{i}^{\prime} \phi_{j}^{\prime}+\phi_{i}^{\prime} \varphi_{j}^{\prime}+\frac{3}{2} y^{\prime} \varphi_{i}^{\prime} \varphi_{j}^{\prime}\right)\right\} d x, \\
& \Gamma_{m i j k}=-\frac{\alpha}{2} \int_{0}^{1} \frac{1}{\rho^{3}}\left\{\phi_{m}^{\prime}\left(\phi_{i}^{\prime} \phi_{j}^{\prime} \phi_{k}^{\prime}+\phi_{i}^{\prime} \varphi_{j}^{\prime} \varphi_{k}^{\prime}\right)+\varphi_{m}^{\prime}\left(\phi_{i}^{\prime} \phi_{j}^{\prime} \varphi_{k}^{\prime}+\varphi_{i}^{\prime} \varphi_{j}^{\prime} \varphi_{k}^{\prime}\right)\right\} d x .
\end{aligned}
$$

\section{ASYMPTOTIC SOLUTION OF NEARLY-TUNED 1:1 INTERNAL RESONANCE}

Periodic solution to Eq. (8) is determined using the method of multiple scales for a weakly non-linear response. The second-order asymptotic analysis is considered, capturing the combined effect of quadratic and cubic nonlinearities. With $\varepsilon$ denoting a small bookkeeping parameter, the damping $\mu_{m}$ and excitation $Z_{m}$ amplitude are ordered such that they balance with the nonlinearity and internal resonance at the same cubic order, i.e., $\mu_{m} \rightarrow \varepsilon^{2} \mu_{m}$ and $Z_{m} \rightarrow \varepsilon^{3} Z_{m}$. In condition of primary external and 1:1 internal resonances, the relevant frequency relationships are quantified as $\Omega=\omega_{s}+\varepsilon^{2} \sigma_{f}$ and $\omega_{s}=\omega_{r}+\varepsilon^{2} \sigma$, in which $\sigma_{f}$ and $\sigma$ are external and internal detuning parameters, respectively. Here, the external resonance of a high-frequency $s$ mode is considered, whereas a low-frequency $r$ mode is internally excited. It is worth noting that around avoidance it is always $\sigma>0$ since $\omega_{s}>\omega_{r}$ (Fig.1b). This is in contrast with cables at crossovers where $\sigma$ may change sign when considering primary resonance of a symmetric mode.

Following Srinil and Rega [20], who consider 1:1 (as well as 2:1) resonant interactions at crossovers and highlight the effects of kinematic condensation on forced vibrations of shallow horizontal cables, the real-valued modulation equations governing the slowly-varying amplitudes $\left(a_{r}, a_{s}\right)$ and relative phases $\left(\gamma_{r}, \gamma_{s}\right)$ of the two interacting hybrid modes, in polar form, read 
$\dot{a}_{r}=-\mu_{r} a_{r}+\frac{K_{1} a_{s}^{3} \sin \Delta}{8 \omega_{r}}+\frac{K_{2} a_{s} a_{r}^{2} \sin \Delta}{8 \omega_{r}}+\frac{K_{3} a_{s}^{2} a_{r} \sin 2 \Delta}{8 \omega_{r}}+\frac{Z_{r} \sin \gamma_{r}}{2 \omega_{r}}$,

$a_{r} \dot{\gamma}_{r}=\left(\sigma_{f}+\sigma\right) a_{r}+\frac{K_{r r} a_{r}^{3}}{8 \omega_{r}}+\frac{K_{r s} a_{r} a_{s}^{2}}{8 \omega_{r}}+\frac{K_{1} a_{s}^{3} \cos \Delta}{8 \omega_{r}}+\frac{3 K_{2} a_{s} a_{r}^{2} \cos \Delta}{8 \omega_{r}}+\frac{K_{3} a_{s}^{2} a_{r} \cos 2 \Delta}{8 \omega_{r}}+\frac{Z_{r} \cos \gamma_{r}}{2 \omega_{r}}$,

$\dot{a}_{s}=-\mu_{s} a_{s}-\frac{K_{2} a_{r}^{3} \sin \Delta}{8 \omega_{s}}-\frac{K_{1} a_{r} a_{s}^{2} \sin \Delta}{8 \omega_{s}}-\frac{K_{3} a_{r}^{2} a_{s} \sin 2 \Delta}{8 \omega_{s}}+\frac{Z_{s} \sin \gamma_{s}}{2 \omega_{s}}$,

$a_{s} \dot{\gamma}_{s}=\sigma_{f} a_{s}+\frac{K_{s s} a_{s}^{3}}{8 \omega_{s}}+\frac{K_{r s} a_{s} a_{r}^{2}}{8 \omega_{s}}+\frac{K_{2} a_{r}^{3} \cos \Delta}{8 \omega_{s}}+\frac{3 K_{1} a_{r} a_{s}^{2} \cos \Delta}{8 \omega_{s}}+\frac{K_{3} a_{r}^{2} a_{s} \cos 2 \Delta}{8 \omega_{s}}+\frac{Z_{s} \cos \gamma_{s}}{2 \omega_{s}}$,

in which $\gamma_{r}=\left(\sigma_{f}+\sigma\right) t-\beta_{r}, \gamma_{s}=\sigma_{f} t-\beta_{s}$, and $\Delta=\gamma_{r}-\gamma_{s}$. In turn, the second-order non-linear interaction coefficients - accounting for infinite-dimensional series of resonant (quadratic/cubic) and non-resonant (quadratic) modes (nonlinearities), governing the internal resonance activation (i.e., $K_{1-3}$ ) and controlling the solution convergence [20,21] - are expressed as:

$$
\begin{aligned}
K_{h h}= & \sum_{m=1}^{\infty}\left[\left(\Lambda_{h h m}+\Lambda_{h m h}\right) \Lambda_{m h h}\left(\frac{2}{\omega_{m}^{2}}+\frac{1}{\omega_{m}^{2}-4 \omega_{h}^{2}}\right)\right]+3 \Gamma_{h h h h}, h=r, s \\
K_{r s}= & \sum_{m=1}^{\infty}\left[\left(\Lambda_{r r m}+\Lambda_{r m r}\right) \frac{2 \Lambda_{m s s}}{\omega_{m}^{2}}+\left(\Lambda_{r s m}+\Lambda_{r m s}\right)\left(\Lambda_{m r s}+\Lambda_{m s r}\right)\left(\frac{1}{\omega_{m}^{2}-\left(\omega_{s}+\omega_{r}\right)^{2}}+\frac{1}{\omega_{m}^{2}-\left(\omega_{s}-\omega_{r}\right)^{2}}\right)\right] \\
& +2\left(\Gamma_{r s s r}+\Gamma_{r s r s}+\Gamma_{r r s s}\right), \\
K_{1}= & \sum_{m=1}^{\infty}\left[\left(\Lambda_{s r m}+\Lambda_{s m r}\right) \frac{\Lambda_{m s s}}{\omega_{m}^{2}-4 \omega_{s}^{2}}+\frac{\left(\Lambda_{s s m}+\Lambda_{s m s}\right)\left(\Lambda_{m r s}+\Lambda_{m s r}\right)}{\omega_{m}^{2}-\left(\omega_{s}-\omega_{r}\right)^{2}}\right]+\Gamma_{s s s r}+\Gamma_{s s r s}+\Gamma_{s r s s}, \\
K_{2}= & \sum_{m=1}^{\infty}\left[\left(\Lambda_{s r m}+\Lambda_{s m r}\right) \Lambda_{m r r}\left(\frac{2}{\omega_{m}^{2}}+\frac{1}{\omega_{m}^{2}-4 \omega_{r}^{2}}\right)\right]+3 \Gamma_{s r r r}, \\
K_{3}= & \sum_{m=1}^{\infty}\left[\left(\Lambda_{s s m}+\Lambda_{s m s}\right) \frac{\Lambda_{m r r}}{\omega_{m}^{2}-4 \omega_{r}^{2}}+\frac{\left(\Lambda_{s r m}+\Lambda_{s m r}\right)\left(\Lambda_{m r s}+\Lambda_{m s r}\right)}{\omega_{m}^{2}-\left(\omega_{s}-\omega_{r}\right)^{2}}\right]+\Gamma_{s r r s}+\Gamma_{s r s r}+\Gamma_{s s r r} .
\end{aligned}
$$

Due to non-linear orthogonality of symmetric/anti-symmetric eigenfunctions, all quadratic and cubic coefficients embedded in $K_{1}$ and $K_{2}$ systematically vanish for crossover cables [20], whereas they exist for avoidance cables involving hybrid (asymmetric) eigenfunctions. In spite of this difference, (nearly tuned) 1:1 resonances are activable near both crossovers and avoidances due to the non-trivial $K_{3}$ and $K_{1-3}$, respectively.

In view of system Eqs.(11)-(14) and (15)-(19), it is worth drawing a general description of possible 1:1 resonant solutions for inclined (avoidance) vs. horizontal (crossover) cables, in 
comparison with other planar internal resonance cases governed by the corresponding modulation equations [20-22]. Depending on the cable parameter $\lambda / \pi$ (Eq. 6), the activated (1:1, $2: 1,3: 1)$ internal resonance and the primary resonance of either a high-frequency $(s)$ or lowfrequency $(r)$ mode, comparison of uncoupled-mode (UC) or coupled-mode (C) solutions for different (non-) crossover/avoidance cables is summarized in Table 1. Whilst both UC and C solutions coexist for 1:1 resonant crossover cables (since $K_{1}, K_{2}=0$ ), only C solution exists for avoidance cables ( in agreement with the analysis of other asymmetric systems [24]. As for 2:1 [20] and 3:1 [22] resonances, both horizontal/inclined cables exhibit UC and C (only $\mathrm{C}$ ) solutions when directly exciting a high-frequency (low-frequency) mode. This implies that 2:1 and 3:1 (1:1) solution kinds do (do not) depend on the mode being directly excited, while they do not (do) depend on the cable geometry being symmetric or asymmetric.

Thus, the modification from crossover to avoidance - as well as from purely symmetric/antisymmetric to hybrid modes - in linear dynamics significantly makes planar 1:1 resonant forced non-linear dynamics of inclined (asymmetric) cables different from horizontal (symmetric) cables. This is a major reason why investigating 1:1 resonant avoidance cables versus 1:1 resonant crossover cables is of theoretical and practical significance.

Mention must be made that if one considers inclined - but still parabolic or symmetric cables exhibiting symmetric/anti-symmetric modes, like done - to the best of our knowledge - in all previous analytical studies [e.g., 2-5], one would obtain the same solution classes as of horizontal cables, which also include the theoretically unrealizable UC solutions. Nonetheless, actual activation of also a non-planar internal resonance to be analyzed with the full 3-D model [10] may occur near avoidances (crossovers). Involving an out-of-plane mode, it would entail a more general classification of non-planar vs. planar solutions than that in Table 1. Yet, this is beyond the scope of this paper, where dealing with only the constrained planar model is sufficient to extract the substantially planar different features of horizontal and inclined cables. 
To deal with stability issue, it is preferable to consider the Cartesian-form modulation equations, in place of Eqs. (11)-(14). By introducing the transformations $a_{i}=\left(p_{i}^{2}+q_{i}^{2}\right)^{1 / 2}$ and $\gamma_{i}=\tan ^{-1}\left(q_{i} / p_{i}\right)$, where $i=r$ or $s$, one arrives at

$$
\begin{aligned}
\dot{p}_{r}= & -\mu_{r} p_{r}-\left(\sigma_{f}+\sigma\right) q_{r}-K_{r r} \frac{\left(p_{r}^{2} q_{r}+q_{r}^{3}\right)}{8 \omega_{r}}-K_{r s} \frac{\left(q_{r} p_{s}^{2}+q_{r} q_{s}^{2}\right)}{8 \omega_{r}}-K_{1} \frac{\left(p_{s}^{2} q_{s}+q_{s}^{3}\right)}{8 \omega_{r}} \\
& -K_{2} \frac{\left(q_{s} p_{r}^{2}+2 p_{s} q_{r} p_{r}+3 q_{s} q_{r}^{2}\right)}{8 \omega_{r}}+K_{3} \frac{\left(p_{s}^{2} q_{r}-2 p_{s} q_{s} p_{r}-q_{s}^{2} q_{r}\right)}{8 \omega_{r}}, \\
\dot{q}_{r}= & -\mu_{r} q_{r}+\left(\sigma_{f}+\sigma\right) p_{r}+K_{r r} \frac{\left(p_{r}^{3}+p_{r} q_{r}^{2}\right)}{8 \omega_{r}}+K_{r s} \frac{\left(p_{r} p_{s}^{2}+p_{r} q_{s}^{2}\right)}{8 \omega_{r}}+K_{1} \frac{\left(p_{s}^{3}+p_{s} q_{s}^{2}\right)}{8 \omega_{r}} \\
& +K_{2} \frac{\left(3 p_{r}^{2} p_{s}+2 p_{r} q_{r} q_{s}+q_{r}^{2} p_{s}\right)}{8 \omega_{r}}+K_{3} \frac{\left(p_{s}^{2} p_{r}+2 p_{s} q_{s} q_{r}-q_{s}^{2} p_{r}\right)}{8 \omega_{r}}+\frac{Z_{r}}{2 \omega_{r}}, \\
\dot{p}_{s}= & -\mu_{s} p_{s}-\sigma_{f} q_{s}-K_{s s} \frac{\left(p_{s}^{2} q_{s}+q_{s}^{3}\right)}{8 \omega_{s}}-K_{r s} \frac{\left(q_{s} p_{r}^{2}+q_{s} q_{r}^{2}\right)}{8 \omega_{s}}-K_{1} \frac{\left(q_{r} p_{s}^{2}+2 p_{s} q_{s} p_{r}+3 q_{r} q_{s}^{2}\right)}{8 \omega_{s}} \\
& -K_{2} \frac{\left(p_{r}^{2} q_{r}+q_{r}^{3}\right)}{8 \omega_{s}}+K_{3} \frac{\left(p_{r}^{2} q_{s}-2 p_{s} q_{r} p_{r}-q_{r}^{2} q_{s}\right)}{8 \omega_{s}}, \\
\dot{q}_{s}= & -\mu_{s} q_{s}+\sigma_{f} p_{s}+K_{s s} \frac{\left(p_{s}^{3}+p_{s} q_{s}^{2}\right)}{8 \omega_{s}}+K_{r s} \frac{\left(p_{s} p_{r}^{2}+p_{s} q_{r}^{2}\right)}{8 \omega_{s}}+K_{1} \frac{\left(3 p_{s}^{2} p_{r}+2 p_{s} q_{s} q_{r}+q_{s}^{2} p_{r}\right)}{8 \omega_{s}} \\
& +K_{2} \frac{\left(p_{r}^{3}+p_{r} q_{r}^{2}\right)}{8 \omega_{s}}+K_{3} \frac{\left(p_{r}^{2} p_{s}+2 p_{r} q_{r} q_{s}-q_{r}^{2} p_{s}\right)}{8 \omega_{s}}+\frac{Z_{s}}{2 \omega_{s}} .
\end{aligned}
$$

Both fixed-point (equilibrium) and dynamic (limit cycles) responses can be evaluated based on this four-dimensional coupled system. Accounting for the multimode expansion, the spacetime varying coupled forced dynamic configurations associated with the $u(J=1)$ or $v(J=2)$ displacement component of a 1:1 resonant inclined cable are expressed, up to second order, as [20]

$$
\begin{aligned}
U^{J}(x, t) & \approx a_{r} \cos \left(\Omega t-\gamma_{r}\right) \zeta_{r}^{J}(x)+a_{s} \cos \left(\Omega t-\gamma_{s}\right) \zeta_{s}^{J}(x)+ \\
& \frac{1}{2}\left\{\begin{array}{l}
a_{s}^{2}\left[\cos \left(2 \Omega t-2 \gamma_{s}\right) \psi_{s s}^{J}(x)+\kappa_{s s}^{J}(x)\right]+a_{r}^{2}\left[\cos \left(2 \Omega t-2 \gamma_{r}\right) \psi_{r r}^{J}(x)+\kappa_{r r}^{J}(x)\right]+ \\
a_{s} a_{r}\left[\cos \left(2 \Omega t-\gamma_{r}-\gamma_{s}\right) \psi_{r s}^{J}(x)+\cos \left(\gamma_{r}-\gamma_{s}\right) \kappa_{r s}^{J}(x)\right]
\end{array}\right\},
\end{aligned}
$$

in which the pertinent second-order coefficients, governing spatial displacement corrections due to quadratic non-linear effects of all resonant/non-resonant modes, are given by

$$
\begin{aligned}
& \psi_{h h}^{J}(x)=\sum_{m=1}^{\infty}\left(\frac{\Lambda_{m h h}}{\omega_{m}^{2}-4 \omega_{h}^{2}} \zeta_{m}^{J}(x)\right), \quad \kappa_{r r}^{J}(x)=\sum_{m=1}^{\infty} \frac{\Lambda_{m h h}}{\omega_{m}^{2}} \zeta_{m}^{J}(x), \quad h=r, s \\
& \psi_{r s}^{J}(x)=\sum_{m=1}^{\infty}\left(\frac{\Lambda_{m r s}+\Lambda_{m s r}}{\omega_{m}^{2}-\left(\omega_{s}+\omega_{r}\right)^{2}} \zeta_{m}^{J}(x)\right), \quad \kappa_{r s}^{J}(x)=\sum_{m=1}^{\infty}\left(\frac{\Lambda_{m r s}+\Lambda_{m s r}}{\omega_{m}^{2}-\left(\omega_{s}-\omega_{r}\right)^{2}} \zeta_{m}^{J}(x)\right) .
\end{aligned}
$$




\section{CABLE PARAMETERS AND INTERACTION COEFFICIENTS}

Because the gap between the two avoided-crossing frequencies in cable spectrum increases at higher avoidance (i.e., at higher sag range) for a fixed inclination $\theta$ (see Fig. $1 \mathrm{~b}$ for $\theta=30^{\circ}$ ), or alternatively, it increases with $\theta$ for a fixed avoidance range $[8,14]$, nearly-tuned 1:1 resonant cables with $\theta=30^{\circ}$ or $45^{\circ}$ at the first two avoidances $(\lambda / \pi \approx 2,4)$ are investigated. Following [20], fixed values of $X_{H}=850 \mathrm{~m}, A \approx 0.1159 \mathrm{~m}^{2}, w_{C} \approx 9.48 \mathrm{kN} / \mathrm{m}$ and $E=1.794 \times 10^{8} \mathrm{kN} / \mathrm{m}^{2}$ are considered. Based on assumed 40 terms of sine-based series in linear dynamics, the parameters $(\alpha, d)$ and frequencies $\left(\omega_{r}, \omega_{s}\right)$, for $\lambda / \pi \approx 2\left(\theta=30^{\circ}, 45^{\circ}\right)$ and $\lambda / \pi \approx 4\left(\theta=30^{\circ}\right)$ are given in Table 2, whereas the corresponding orthonormalized $(u, v)$ shape functions are depicted in Fig.2.

The monochrome bar charts in Fig.3 schematically show percent contributions of each resonant/non-resonant mode to the second-order quadratic coefficients embedded in $K_{r r}, K_{s s}, K_{r s}$, $K_{1}, K_{2}$ and $K_{3}$ (Eqs. 15-19). By accounting for the first 10 and 15 modes through the Galerkin projection (Eq. 7), Figures $3 \mathrm{a}$ and $3 \mathrm{~b}(3 \mathrm{c})$ correspond to the first (second) avoidance with $\theta=30^{\circ}$ and $45^{\circ}\left(\theta=30^{\circ}\right)$, respectively. In turn, the ensuing effective coefficients accounting for overall contributions of quadratic and cubic nonlinearities are reported in Table 3.

Even though all inclined cables have small values of sag-to-span ratio $d$, less than 1:8 [12], Figure 2 reveals qualitative features of resonant hybrid (asymmetric) modes since the cubic asymmetric static configuration (Eq.5) has been considered. It is also seen that the $u(v)$ modal component in the fixed Cartesian co-ordinate system plays an increasing (decreasing) role as $\theta$ increases for a fixed $\lambda / \pi=2$. In view of Fig.3, all charts highlight significant contributions of both resonant (encircled) and non-resonant (lower and/or higher-order) modes for 1:1 resonant avoidance cables, confirming the companion analysis of $1: 1$ (2:1) resonant crossover (noncrossover/non-avoidance) cables [20] ([11]).

As a matter of fact, each quadratic modal contribution, which gives rise to either a positive or negative coefficient correction, depends on overall contributions of (i) elasto-geometric 
parameter and static configuration, (ii) modal characteristics of horizontal/vertical displacements (e.g., Fig.2), and (iii) system frequency commensurability [11]. Some or all of these factors also affect the outcomes of cubic coefficients embedded in Eqs.(15)-(19) and second-order shape functions given by Eq. (25). For $\theta=30^{\circ}$, the second-avoidance cable which involves higherorder $(r=3, s=4)$ hybrid modes requires a higher order modal truncation (up to $m \approx 9$ ) in Fig.3c, than the first-avoidance cable in Fig.3a (up to $m \approx 5$ ), due to the increased sag effect [11].

In contrast with a 1:1 resonant first-crossover cable whose non-trivial contributions of nonresonant anti-symmetric modes $(m=4,6, \ldots)$ to $K_{r s}$ or $K_{3}$ are negligible [20], the 1:1 resonant first-avoidance cables in Figs.3a $\left(\theta=30^{\circ}\right)$ and $3 \mathrm{~b}\left(\theta=45^{\circ}\right)$ reveal meaningful contributions of the $m=4$ mode to $K_{r s}$, though with a different sign. This occurs, on the one side, due to the high modal density of asymmetric resonant/non-resonant shape functions entering $K_{r s}$ through, e.g., $\Lambda_{r s m}$ (see Eq.9); on the other side, due to the associated frequency factor, whose order of magnitude is, in general, $O\left(10^{-2}\right)-O\left(10^{-4}\right)$, becoming as higher as $O(1)$ for $1 /\left(\omega_{m}^{2}-\left(\omega_{s}+\omega_{r}\right)^{2}\right)$ with $m=4$, since $\omega_{m} \approx 2 \omega_{s} \approx 2 \omega_{r}$. The latter also warns about possible activation of a multiple planar internal resonance [11]. Yet, regardless of the frequency factor, the solely effects of asymmetric modal contributions of non-resonant modes may still play a remarkable role: see the $K_{3}$ contributions of $3^{\text {rd }}$ and $5^{\text {th }}$ modes in both Figs. 3 a and $3 b$, as well as contributions of $2^{\text {nd }}, 5^{\text {th }}$ and higher-order modes in Fig.3c for the second-avoidance cable.

In Table 3, overall comparison of the effective coefficients reveals quantitative as well as qualitative differences in the non-linear contributions (values and/or sign) to different avoidance inclined cables. This circumstance affects in a variable way the ensuing non-linear dynamics and stability under given control parameters, as highlighted in the following. 


\section{NON-LINEAR DYNAMIC RESPONSES AND PHENOMENA}

Using the continuation technique [23], the non-linear forced responses of nearly-tuned 1:1 resonant inclined cables are now obtained based on Eqs. (20)-(23). The fixed-point solution is evaluated by the Newton-Raphson method and the dynamic (limit cycle) solution is evaluated by the multiple shooting application. Upon quasi-statically varying a control parameter $\left(\sigma_{f}, F\right)$ within a small incremental and adaptive step-size, the stability of fixed points is examined based on eigenvalues of the relevant $4 \times 4$ Jacobian matrix, whereas that of limit cycles is based on Floquet multipliers. Depending on initial conditions, the dynamic solution is investigated by direct numerical integrations of Eqs.(20)-(23). Here, we utilize the fourth-order Runge-Kutta scheme with stable time step equal to .0001 and other tools including phase-plane projections, Fourier power spectral density (PSD) and Poincarè maps (sampled at each forcing period) to characterize specific responses after decayed transient states. Note that the numerical simulation is herein solely aimed at characterizing dynamic solutions upon continuation bifurcation predictions. Should one be interested in validating the reduced-order models embedded in the perturbation solution, simulations should affect the original partial-differential Eqs.(3)-(4) governing the actual non-linear system.

As described in Sect. 3, only coupled $a_{s}$ - $a_{r}$ (directly-indirectly excited) mode solution exists in 1:1 resonant avoidance cables, and $\sigma>0$ is realized. Throughout the analysis, small damping coefficients $c$ are chosen such that $\mu_{r}=.005$ and $\mu_{s}=.006$. Solid lines indicate stable fixed points, whereas dashed or dotted lines indicate unstable fixed points due to a saddle-node (SN) or a Hopf (HF) bifurcation, respectively. The latter plays a crucial role in the onset of periodic, quasiperiodic and chaotic responses. Filled (open) symbols, e.g., circles, triangles or squares, indicate stable (unstable) limit cycles of amplitudes and phases.

\subsection{Bifurcation Analysis with Influence of Parameters}


With $\theta=30^{\circ}, \sigma=.04$ and $F=.002$, Fig. 4 compares frequency-response curves of $a_{r}$ and $a_{s}$ amplitudes for first- (a-d) and second-avoidance (e-f) cables. To illustrate the effect of canceling the indirect forcing term, we put $\Upsilon_{r}=0$ in Figs.4c and $4 d$, with respect to others that fully account for non-trivial $\Upsilon_{r}$ and $\Upsilon_{s}$. Overall, the responses exhibit two coupled-mode solutions isolated from each other: the main one (B1) entirely spanning the frequency $\sigma_{f}$ range with predominant $a_{r}$ amplitudes, whereas the other one (B2) exhibiting the so-called frequency island phenomenon and coexisting in a particular range left to the zero- $\sigma_{f}$ axis, with predominant $a_{s}$ amplitudes. This feature occurs regardless of the actual differences in values and/or sign of nonlinear coefficients between first- and second-avoidance cables (Table 3), and of whether $\Upsilon_{r}$ being zero. From a stability viewpoint, it is worth distinguishing that, due to SN bifurcations, the unstable portions of B1 branch have one positive real eigenvalue of the Jacobian matrix, whereas those of B2 branch may have one or two positive real eigenvalues.

For first avoidance cable with non-zero $\Upsilon_{r}$ and $\Upsilon_{s}$ (Figs.4a, 4b), up to 7 (three stable and four unstable) fixed points may coexist and a pair of $\operatorname{Hopf}\left(\mathrm{HF}_{1,2}\right.$ or $\left.\mathrm{HF}_{3,4}\right)$ bifurcations occur in each branch, though with a marginal range of ensuing dynamic solutions. These dynamic characteristics differ from those of the corresponding 1:1 resonant crossover cable [20] where the B1 and B2 branches merge together, giving rise to both uncoupled- and coupled-mode responses, with the latter being originated from the former via pitchfork bifurcations and with Hopf bifurcations being found only in the coupled-mode branch. Considering $\Upsilon_{r}=0$ in Figs.4c and $4 \mathrm{~d}$ mainly affects the jump (hysteresis) phenomena in B1 branches, with the SN points being shifted with respect to those in Figs.4a and $4 \mathrm{~b}$ when sweeping $\sigma_{f}$ up or down. Moreover, up to 5 (two stable and three unstable) fixed points may coexist. It is worth mentioning that coupled-mode responses and frequency islands have recently been documented in [24], which considered 1:1 resonant imperfect beams at first avoidance with primary resonance of a low-frequency mode and surprisingly accounted for some cases with $\Upsilon_{s}=0$ and a physically unrealistic value $(\sigma=0)$ 
of internal detuning. Therein, only a single Hopf bifurcation has been shown to occur. For the sake of completeness, we consider both $\Upsilon_{r}$ and $\Upsilon_{s} \neq 0$ in the subsequent analysis.

For second avoidance cable (Figs.4e and 4f), overall $a_{r}$ and $a_{s}$ responses become smaller and the B2 branch considerably shifts to the left boundary of the considered $\sigma_{f}$ range: this implies that the hybrid modal interaction role is solely played by the main B1 branch around nearly perfect primary resonance.

Keeping all parameters unchanged, significant qualitative differences occur in Figs.5a and $5 \mathrm{~b}$ for first-avoidance cable with higher $\theta=45^{\circ}$, with respect to Figs.4a and $4 \mathrm{~b}$. In particular, overall jump phenomena occur through turning points in a smaller $\sigma_{f}$ range $(-.075, .075)$ and there are 3 Hopf bifurcations: both $\mathrm{HF}_{1,2}$ are still found in the main $\mathrm{B} 1$ branch, whereas a single $\mathrm{HF}_{3}$ is found in the B2 island along with a connection of unstable foci and saddles (having two positive real eigenvalues). Both isolated solutions entirely overlap each other, with meaningful ranges of two unstable foci coexisting in a particular $\sigma_{f}$ range nearly centered at the zero- $\sigma_{f}$ axis. Depending on initial conditions, in this case up to 5 (three stable and two unstable or two stable and three unstable) fixed points may coexist.

To gain insight into dynamic interactions, the solution emerged from each HF point is traced out in Figs.5c $\left(a_{r}\right)$ and $5 \mathrm{~d}\left(a_{s}\right)$, which reveal individual routes of continuation results. All $\mathrm{HF}_{1-3}$ bifurcations occurring at $\sigma_{f} \approx-.01176, .02837,-.00105$, respectively, are of super-critical type because the born limit cycles are stable. Interestingly, all dynamic solution branches highlight how they lose stability via period-doubling $\left(\mathrm{PD}_{1-3}\right)$ bifurcations (at $\sigma_{f} \approx-.0074, .0236, .0027$ ), each of which having one critical Floquet multiplier that crosses the unit circle at -1 [23]. Such PD bifurcations pave the way to quasi-periodic or chaotic oscillations, depending on given $\sigma_{f}$ values and initial conditions (see Sect. 5.2).

With reference to Figs. 4 and $5(a, b)$, it is now of interest to obtain the responses by varying $F$. To also appreciate the internal detuning $\sigma$ effect, we consider $\sigma=.04$ and .08 for $\theta=30^{\circ}$. 
With $\sigma_{f}=0$, the obtained $a_{r}$ and $a_{s}$ amplitudes are displayed in Figs.6a and 6b, respectively. Again, it is found that two isolated (B1, B2) branches take place in all cases, with $a_{r}\left(a_{s}\right)$ amplitudes predominating in the B1 (B2) branch. All fixed-point B1 solutions lose and gain stability via $\mathrm{HF}$ bifurcations at low $\left(\mathrm{HF}_{1}\right)$ and higher $\left(\mathrm{HF}_{2}\right)$ forcing $F$ amplitude, respectively. As regards $\mathrm{B} 2$ branch, there exists a single $\mathrm{HF}$ for cable with $\theta=45^{\circ}$, in accordance with the corresponding B2 curves in Figs.5a and 5b, whereas there is no HF bifurcation for cable with $\theta=$ $30^{\circ}$, which, instead, experiences SN points for all $\sigma$ cases. As $\sigma$ increases for $\theta=30^{\circ}$, the range of B1 unstable foci substantially increases and the B2 fixed points take place at a higher $F$ amplitude. The latter implies that, for perfect external resonance, only the B1 solution does exist in the low $F$ range for a greater $\sigma$.

By focusing on the cable with $\theta=30^{\circ}$, Figs.7a $\left(a_{r}\right)$ and $7 \mathrm{~b}\left(a_{s}\right)$ highlight the $\sigma$ effect on B1 dynamic solution starting from the two HF points in Figs.6a and 6b. Qualitative differences regarding the solution stability occur when increasing $\sigma$. For $\sigma=.04$, the $\mathrm{HF}_{2}\left(\mathrm{HF}_{1}\right)$ at $F$ $\approx .002446(.000486)$ is of sub-critical (super-critical) type, and the relevant stable solution (triangle) loses stability via cyclic-fold $(\mathrm{CF})$ bifurcation when increasing $F(\approx .004437)$ Thus, the dynamic solution jumps to the associated stable fixed-point in Fig.6. For $\sigma \approx .08$, both the $\mathrm{HF}_{1}$ and $\mathrm{HF}_{2}$ shifted to higher $F(\approx .000975$ and .007571$)$ are of super-critical type. The corresponding dynamic solution (circle) branches are isolated from each other: the stable one emerged from $\mathrm{HF}_{2}$ terminating shortly after decreasing $F(\approx .007341)$, whereas the other one emerged from $\mathrm{HF}_{1}$ losing stability via $\mathrm{PD}$ bifurcation at $F \approx .002013$. When further increasing $F$, the latter undergoes a reverse PD bifurcation at $F \approx .006378$, where the dynamics is re-stabilized, and the continuing response grows monotonously afterwards, with $a_{r}$ increasing by a rate greater than $a_{s}$. Thus, for both $\sigma$ values, one observes simultaneous existence of stable fixed-point and periodic response for the same B1 solution, apart from a transition to chaos possibly existing between the two PD points. 


\subsection{Periodic, Quasi-Periodic and Chaotic Responses}

Direct numerical integration results - validating the continuation prediction and showing rich non-linear dynamics after HF and PD bifurcations - are now presented. Along the branch starting from $\mathrm{HF}_{2}$ in Figs.5c and 5d, a sequence of PD responses leading to chaos is highlighted by the $p_{r}-q_{r}$ phase portraits in Fig. 8 , where $\sigma_{f}$ is slowly decreased $\left(\sigma_{f}=.025 \rightarrow .02235\right)$ with fixed initial conditions corresponding to the $\mathrm{PD}_{2}$ solution at $\sigma_{f}=.02357$, i.e., $\left(p_{r}, q_{r}, p_{s}, q_{s}\right)=(-.0013251$, $-.0002848, .00113897, .00077433)$. It is seen that as the limit cycle (Fig.8a) experiences twocycle (Fig.8b), four-cycle (Fig.8c) and multiple-cycle (Fig.8d) closed loops generated by consecutive PD bifurcations, the last trajectory undergoes a chaotically-modulated response at $\sigma_{f}$ $=.0225$ (Fig.8e), thereby giving rise to aperiodic time histories. Further slightly decreasing $\sigma_{f}$ (Fig.8f), transient chaos appears and then the orbit undergoes a likely boundary crisis, where the chaotic set is destroyed and the post-bifurcation state suddenly escapes to the bounded fixedpoint B1 solution (Figs.5a, 5b).

A route to chaos via $\mathrm{PD}$ is also detected when continuing the solution from $\mathrm{HF}_{1}$ with a slow increment in $\sigma_{f}$. By assigning $\left(p_{r}, q_{r}, p_{s}, q_{s}\right)=(-.0007886,-.0002589, .0009279, .0006741)$ of $\mathrm{PD}_{1}$ solution $\left(\sigma_{f}=-.00735\right)$ as initial conditions, the $p_{r}-q_{r}$ and $p_{s}-q_{s}$ phase planes which exhibit a funnel-shaped chaotic attractor [25] at $\sigma_{f}=-.004812$ are shown in Figs.9a and 9b. The associated Poincarè $a_{r}-a_{s}$ section, calculated for about 20,000 periods, reveals a collection of points spreading out over the limited region in Fig.9c. Increasing $\sigma_{f}$ thereafter, a crisis occurs at $\sigma_{f}=$ -.004312 and the resulting response ends up with a stable fixed point of the overlapped B2 branch in Figs.5a and 5b.

Qualitative differences occur regarding the route to chaos when continuing the dynamic solution from $\mathrm{HF}_{3}$ of $\mathrm{B} 2$ branch in Figs.5c and 5d. Since neither fixed point nor periodic stable solutions take place in the region between $\mathrm{PD}_{3}\left(\sigma_{f}=.00269\right)$ and the saddle node $\left(\mathrm{SN}^{*}, \sigma_{f}\right.$ $=.01032)$ of B1 branch, one would expect either quasi-periodic or aperiodic response, depending 
on initial conditions. By initiating with the $\mathrm{PD}_{3}$ solution $\left(p_{r}, q_{r}, p_{s}, q_{s}\right)=(.0001610, .0005058$, $-.0027938, .0014901)$ and slowly increasing $\sigma_{f}$ towards $\sigma_{f} \approx .0035$, the ensuing non-linear time $(p-q)$ histories exhibit intermittently chaotic outbursts, as shown in Fig.10. Thus, the $\mathrm{PD}_{3}$ bifurcation may be of sub-critical type, and the ensuing intermittency may be of type III (on-off intermittency) [25]. Applying the same initial conditions and varying $\sigma_{f}$ further, the non-linear dynamics may be quasi-periodic as well as chaotic. The occurrence of quasi-periodic motion is exemplified by the time histories and Poincarè map of $a_{r}-a_{s}$ amplitudes in Fig. 11 for $\sigma_{f}=.006$. Figures 11a and 11b highlight multi-frequency responses, whereas Fig.11c exhibits a closed-loop map. Of course, when $\sigma_{f}$ meets a critical value, a crisis finally occurs $\left(\sigma_{f} \approx .011\right)$ and the response jumps to the stable fixed point of B1 branch. Thus, the observed sudden changes in non-linear response via crises may lead to switching between dynamic and equilibrium solutions of two overlapping branches.

Examples of PSD corresponding to $q_{s}$ responses in Figs.8d, 8e, 9 and 11 are shown in Figs.12a-d, respectively. The PSD of Fig.12a illustrates how, when period doubling takes place, the dominant sharp peak and its sub-harmonics simultaneously occur along with their integer multiples, justifying the multiple closed-loop orbit in Fig.8d. On the contrary, due to chaoticallymodulated oscillations (Figs.8e, 9), the PSDs of Figs.12b and 12c highlight a major spike associated with the excitation frequency and many side-banding components distributed continuously over a broad frequency band with high density. Differently, the PSD of Fig.12d exhibits many harmonics whose frequencies are not commensurate in perfectly integer ratios, e.g., one being 1.33 between the first two dominant (high- and low-frequency) peaks. This confirms how the response in Fig. 11 is quasi-periodic.

Dynamic insight into 1:1 resonant interactions are now discussed through the force-response diagrams in Fig.7. No chaotic motion is found when varying $F$ for $\sigma=.04$. In other words, either periodic or quasi-periodic response with variable time-modulated amplitudes and phases is attained. On the other hand, for $\sigma=.08$, the chaotic vibration is found in the range between the 
two PD bifurcations. With initial conditions of the first PD $(F=.002)$, i.e., $\left(p_{r}, q_{r}, p_{s}, q_{s}\right)=$ $(-.0001368,-.0000259, .0006552, .0005225)$ and with increasing $F$ from .0015 to .00244 , the relevant phase-plane plots of $p_{r}-p_{s}$ projection are shown in Figs.13a-13d, which exhibit a cascade of seemingly PD bifurcations (as checked via PSDs) together with a considerable increment in size of the trajectories. The chaotic response takes place at $F \approx .0025$ and dominates over a wide $F$ range. As an example, Fig. $13 \mathrm{e}$ shows the chaotic response at $F=.003$ whose non-periodic amplitudes are significantly large. Thus, one must worry about substantial induced dynamic tensions in the cable. When increasing $F$, another crisis may occur prior to $F=.006378$ corresponding to the second PD, with the chaotic response becoming quasi-periodic, see, e.g. Fig. $13 \mathrm{f}$ for $F=.0055$, since no stable fixed point coexists (Fig.6).

The coexistence of slightly different attractors at a given value of $F$ is highlighted in Fig.14 through $p_{s}-q_{s}$ phase planes (Figs.14a, 14b) and ensuing Poincarè $a_{r}-a_{s}$ sections (Figs.14c, 14d). With $F=.007$, two sets (I, II) of initial conditions $\left(p_{r}, q_{r}, p_{s}, q_{s}\right)$ are considered in numerical integrations for the cable in Fig.7 with $\sigma=.08$ : set I corresponding to the unstable B1 fixed point at $F=.006(-.0026334, .0000658, .0005957, .0001496)$ in Figs.14a and 14c, and set II corresponding to the stable periodic solution from $\mathrm{HF}_{1}$ at $F=.0074(-.0012781$, $-.0001358, .0000670, .0009374)$ in Figs.14b and 14d. It is shown that both the extent and duration of amplitude modulation are different between Figs.14a and 14b. Whilst the Poincarè map of the former (Fig.14c) exhibits a three closed-loop orbit, that of the latter (Fig.14d) provides a single closed-loop one. The relevant PSD of $q_{s}$ in Figs.15a (I) and 15b (II) show two dominantly incommensurate frequencies, highlighting how they are both quasi-periodic. Yet, the former reveals also the sub-harmonic components of the three closed-loop orbit in Fig.14c.

\subsection{Non-linear Dynamic Displacements and Tensions}

Space-time distributions governing steady and chaotically-modulated dynamic displacements and tensions due to 1:1 resonant interactions are now highlighted. Depending on the obtained 
amplitudes $\left(a_{r}, a_{s}\right)$ and phases $\left(\gamma_{r}, \gamma_{s}\right)$, the second-order $(u, v)$ coupled dynamic deflections are constructed through Eq. (24), whereas the relevant dynamic tensions $\left(T_{d}=E A e_{d}\right)$ are evaluated based on displacement gradients through the strain $e_{d}$ in Eq. (2). The $T_{d}$ value is normalized with respect to the associated maximum static tension $T_{H}$.

The first comparison of steady responses is illustrated in Fig.16 for first-avoidance cables having different stable fixed points. With $\sigma=.04, F=.002$, and $\sigma_{f}=-.02$, the $u, v$ and $T_{d} / T_{H}$ responses of B1 solution of cable with $\theta=30^{\circ}$ (Figs.4a and 4b) are visualized in Figs.16a-16c, whereas those of $\mathrm{B} 1$ and $\mathrm{B} 2$ solution of cable with $\theta=45^{\circ}$ (Figs.5a and 5b) are visualized in Figs.16d-16f and 16g-16i, respectively, over a half period of harmonic excitation. Here, empty (filled) circles indicate the initial (half-period) responses. With regard to the B1 solution, the cable responses with $\theta=30^{\circ}$ (Figs.16a, 16b) and $45^{\circ}$ (Figs.16d, 16e) qualitatively agree for the $u$ and $v$ asymmetric profiles exhibiting predominance of the indirectly-excited $r$ mode (see Fig.2) according to the fact that the $a_{r}$ amplitudes $(.00210, .00134)$ are significantly greater - by an order of magnitude - than the $a_{s}$ amplitudes $(.00019, .000475)$ for both $\theta\left(30^{\circ}, 45^{\circ}\right)$. In addition, there is no sign difference in the corresponding phases of the two cables: $\left(\gamma_{r}, \gamma_{s}\right) \approx(-.12268$, $1.21083)$ for $\theta=30^{\circ}$, whereas $\left(\gamma_{r}, \gamma_{s}\right) \approx(-.29196, .30159)$ for $\theta=45^{\circ}$. As the dominant $a_{r}$ decreases as $\theta$ increases, the spatially asymmetric feature of $T_{d} / T_{H}$ responses due to strain variation effect is more significant with $\theta=30^{\circ}$ than with $\theta=45^{\circ}$, and also entails larger time excursions of $T_{d} / T_{H}$ magnitudes.

With respect to $\mathrm{B} 1$ responses, the associated B2 (frequency-island) responses of cable with $\theta$ $=45^{\circ}$ in Figs.16g and $16 \mathrm{~h}$ exhibit a sign difference in relative phases $\left(\gamma_{r} \approx-.79040, \gamma_{s} \approx-.69213\right)$ and the predominance of the directly-excited $s$ mode because of the $a_{s}$ amplitude (.00347) being now much greater than the $a_{r}$ one (.0006). Accordingly, the second-order spatial corrections from resonant and non-resonant (higher-order) modes are seen to be significant due to quadratic nonlinear effects (Sect. 4), thereby leading to essentially multi-mode responses. Apart from the 
induced $T_{d} / T_{H}$ responses being greater, both spatial and temporal variations of tensile/compressive forces are considerably important.

Next, the comparison of two coexisting periodic and chaotic responses is shown in Fig.17 for cable with $\theta=45^{\circ}$. With $\sigma=.04, F=.002$, and $\sigma_{f}=.0225$, either the steady-state amplitudes (phases) of stable B1 solution (Figs.5a and 5b) or the chaotically modulated amplitudes (phases) based on numerical integrations of modulation equations (Fig.8e) are considered. The associated periodic and non-periodic $\left(u, v, T_{d} / T_{H}\right)$ responses are depicted in Figs.17a-c and $17 \mathrm{~d}-\mathrm{f}$, respectively, the latter being recorded during the interval $t \approx 211.2-216.2$ (about 5 periods of excitation) with 0.1 being the time increment.

Unlike the $\theta=45^{\circ}$ case with $\sigma_{f}=-.02$ (Figs.16d, 16e), the steady $u$ and $v$ responses with $\sigma_{f}$ $=.0225$ (Figs.17a, 17b) exhibit the predominant $s$-mode profiles because $a_{s}(.001156)$ is greater than $a_{r}(.000252)$, with $\gamma_{s} \approx-.23094$ vs. $\gamma_{r} \approx-.05332$. This highlights how, depending on the control parameter, one given solution (e.g., B1) may, of course, be dominated by either the directly- or indirectly-excited mode. On the contrary, the multi-modal asymmetric spatial responses become evident when the cable experiences chaos, as shown in Figs.17d and 17e. Since the varying $a_{r}, a_{s}$ amplitudes may have the same order of magnitude (e.g., $a_{r} \approx .002121$, $a_{s} \approx .001437$ at $t \approx 212.3$ ), from a modal superimposition viewpoint contributions from the two resonant modes are comparatively important, with also meaningful second-order spatial corrections from all retained modes. Consequently, in Fig.17f, the induced dynamic tensile/compressive forces are outstandingly increased, with the associated non-periodic nonuniform spatial distribution, whereas in Fig.17c, the small-amplitude responses show rather spatially-uniform dynamic stresses. Thus, in cable design, one has to worry about possibly chaotic 1:1 resonant oscillations. 


\section{CONCLUSIONS}

Non-linear planar forced oscillations of elastic sagged inclined cables due to 1:1 internal resonance of hybrid modes at avoidances have been investigated. The cable is subjected to a uniformly-distributed vertical harmonic excitation with primary resonance of a high-frequency mode. Approximate non-linear equations of motion account for the asymmetry of inclined sagged configurations, overall displacement coupling and dynamic extensibility. Based on a multimode expansion and second-order multiple scales solution, analysis of modulation equations governing the planar $1: 1$ - vs. $2: 1$ or $3: 1$ - resonant interactions of horizontal/inclined cables allows us to highlight how the modification from crossover to avoidance-as well as from purely symmetric/anti-symmetric to hybrid modes - in linear dynamics significantly makes 1:1 resonant forced non-linear dynamics of inclined (asymmetric) cables different from horizontal (symmetric) cables. In turn, the modal analysis of quadratic non-linear coefficients highlights significant contributions of both resonant and non-resonant (asymmetric) modes to 1:1 resonant asymptotic solutions.

Bifurcation analysis provides information about a multiplicity of (stable/unstable) equilibrium and periodic solutions, as well as meaningful transitions from periodic to quasiperiodic and chaotic responses. Essentially, isolated coupled-mode solution branches coexist with frequency islands, experiencing saddle-node and Hopf bifurcations. Limit cycles may undergo cyclic-fold as well as direct/reverse period-doubling bifurcations. Overall results highlight the influence of cable inclination and internal resonance detuning on non-linear dynamic behavior of avoidance cables and the effect of cable sag at higher avoidance. Direct numerical integrations validate the continuation prediction and characterize post-bifurcation dynamics. Depending on system control parameters and initial conditions, a whole cornucopia of non-linear phenomena are observed: viz., sequences of period-doubling bifurcations leading to chaos, funnel-shaped chaos, on-off intermittency mechanism, and sudden switching of solutions via boundary crises, with the competing effect of dynamic solutions also taken into account. 
Depending on resonant amplitudes, non-linear dynamic displacements and tensions illustrate the predominance of either the indirectly- or directly-excited asymmetric mode, along with some higher-order spatial corrections. Spatial dynamic deflections associated with chaotically resonant vibrations exhibit multi-mode features with significant time-varying amplitudes. As a result, the chaotic dynamics are endowed with remarkable asymmetry of spatially non-uniform, strongly time-varying, tensile/compressive dynamic tensions.

Acknowledgement: Dr. Narakorn Srinil is deeply indebted to the support from the University of Rome 'La Sapienza', Italy, through a Postdoctoral Fellowship.

\section{References}

1. Rega, G., 'Nonlinear dynamics of suspended cables. Part I: Modeling and analysis; Part II: Deterministic phenomena', ASME Applied Mechanics Reviews 57, 2004, 443-514.

2. Cai, Y., Chen, S.S., 'Dynamics of elastic cable under parametric and external resonances', ASCE Journal of Engineering Mechanics 120, 1994, 1786-1802.

3. Pinto Da Costa, A., Martins, J.A.C., Branco, F., Lilien, J.L., 'Oscillations of bridge stay cables induced by periodic motions of deck and/or towers', ASCE Journal of Structural Engineering 122, 1996, 613-622.

4. Nielsen, S.R.K., Kirkegaard, P.H., 'Super and combinatorial harmonic response of flexible elastic cables with small sag', Journal of Sound and Vibration 251, 2002, 79-102.

5. Zhao, Y.Y., Wang, L.H., Chen, D.L., Jiang, L.Z., 'Non-linear dynamic analysis of the twodimensional simplified model of an elastic cable', Journal of Sound and Vibration 255, 2002, 43-59.

6. Caswita, Van Der Burgh, A.H.P., 'Combined parametrical transverse and in-plane harmonic response of an inclined stretched string' Journal of Sound and Vibration 267, 2003, 913-931.

7. Takahashi, K., Konishi, Y., 'Non-linear vibrations of cables in three dimensions, Part I: Non-linear free vibrations', Journal of Sound and Vibration 118, 1987, 69-84.

8. Srinil, N., Rega, G., Chucheepsakul, S., 'Large amplitude three-dimensional free vibrations of inclined sagged elastic cables', Nonlinear Dynamics 33, 2003, 129-154.

9. Srinil, N., Rega, G., Chucheepsakul, S., 'Three-dimensional nonlinear coupling and dynamic tension in the large amplitude free vibrations of arbitrarily sagged cables', Journal of Sound and Vibration 269, 2004, 823-852.

10. Srinil, N., Rega, G., Chucheepsakul, S., 'Two-to-one resonant multi-modal dynamics of horizontal/inclined cables. Part I: Theoretical formulation and model validation', Nonlinear Dynamics 48, 2007, 231-252. 
11. Srinil, N., Rega, G., 'Two-to-one resonant multi-modal dynamics of horizontal/inclined cables. Part II: Internal resonance activation, reduced-order models and nonlinear normal modes', Nonlinear Dynamics 48, 2007, 253-274.

12. Irvine, H.M., Cable Structures, MIT Press, Cambridge, 1981.

13. Triantafyllou, M.S., 'The dynamics of taut inclined cables', Quartery Journal of Mechanics and Applied Mathematics 37, 1984, 421-440.

14. Triantafyllou, M.S., Grinfogel, L., 'Natural frequencies and modes of inclined cables', ASCE Journal of Structural Engineering 112, 1986, 139-148.

15. Perkins, N.C., Mote Jr., C.D., 'Comments of curve veering in eigenvalue problems', Journal of Sound and Vibration 106, 1986, 451-463.

16. Russell, J.C., Lardner, T.J., 'Experimental determination of frequencies and tension for elastic cables', ASCE Journal of Engineering Mechanics 124, 1998, 1067-1072.

17. Srinil, N., 'Large-amplitude three-dimensional dynamic analysis of arbitrarily inclined sagged extensible cables', Ph.D. Dissertation, King Mongkut's University of Technology Thonburi, Bangkok, Thailand, 2004, 1-209.

18. Xu, Y.L., Yu, Z., 'Vibration of inclined sag cables with oil dampers in cable-stayed bridges', ASCE Journal of Bridge Engineering 3, 1998, 194-203.

19. Bendiksen, O.O., 'Localization phenomena in structural dynamics', Chaos, Solitons and Fractals 11, 2000, 1621-1660.

20. Srinil, N., Rega, G., 'The effects of kinematic condensation on internally resonant forced vibrations of shallow horizontal cables', International Journal of Non-linear Mechanics, 2007, doi:10.1016/j.ijnonlinmec.2006.09.005.

21. Lacarbonara, W., Rega, G., Nayfeh, A.H., 'Resonant nonlinear normal modes. Part I: Analytical treatment for structural one-dimensional systems', International Journal of Non-linear Mechanics $38,2003,851-872$.

22. Zhao, Y., Wang, L., 'On the symmetric modal interaction of the suspended cable: Three-to-one internal resonance', Journal of Sound and Vibration 294, 2006, 1073-1093.

23. Seydel, R., Practical Bifurcation and Stability Analysis, Springer, New York, 1994.

24. Lacarbonara, W., Arafat, H.N., Nayfeh, A.H., 'Non-linear interactions in imperfect beams at veering', International Journal of Non-linear Mechanics 40, 2005, 987-1003.

25. Nayfeh, A.H., Balachandran, B., Applied Nonlinear Dynamics, Wiley, New York, 1995. 
Figure

\section{List of Figures}

$1 \quad$ (a) Schematic model of a sagged inclined cable.

(b) Planar frequency spectrum and avoidance phenomena of inclined cable with $\theta=30^{\circ}$.

2 Low- and high-frequency $(r, s)$ modal shapes of $u$ and $v$ displacements: Solid (dashed, dotted) lines denote $\theta=30^{\circ}$ and $\lambda / \pi \approx 2\left(\theta=45^{\circ}\right.$ and $\lambda / \pi \approx 2, \theta=30^{\circ}$ and $\left.\lambda / \pi \approx 4\right)$.

3 Percent modal contributions to second-order quadratic coefficients embedded in $K_{r r}, K_{s s}, K_{r s}, K_{1}$, $K_{2}$ and $K_{3}$ : (a) $\theta=30^{\circ}, \lambda / \pi \approx 2$; (b) $\theta=45^{\circ}, \lambda / \pi \approx 2$; (c) $\theta=30^{\circ}, \lambda / \pi \approx 4$.

4 Frequency-response diagrams and bifurcations of inclined cables with $\theta=30^{\circ}, \sigma=.04, F=.002$ : (a)-(b) $\lambda / \pi \approx 2$; (c)-(d) $\lambda / \pi \approx 2$ but with $\Upsilon_{r}=0$; (e)-(f) $\lambda / \pi \approx 4$.

5 Frequency-response diagrams and bifurcations of inclined cables with $\theta=45^{\circ}, \sigma=.04, F=.002$ : (a)-(b) fixed-point solution; (c)-(d) dynamic solution.

6 Force-response diagrams and bifurcations of inclined cables with $\sigma_{f}=0$ : influence of $\theta$ and $\sigma$.

7 Dynamic solutions and bifurcations along force-response diagrams of inclined cable with $\theta=$ $30^{\circ}, \sigma_{f}=0$ : triangles (circles) denote $\sigma=.04(\sigma=.08)$.

8 Phase portraits showing the transition from periodically to chaotically-modulated dynamic response and the boundary crisis of inclined cable in Fig.5 (from $\mathrm{HF}_{2}$ ).

9 Phase portraits and Poincarè map showing chaotically funnel-shaped dynamic response of inclined cable in Fig.5 with $\sigma_{f}=-.004812$ (from $\mathrm{HF}_{1}$ ).

10 Time response histories showing the intermittently chaotic response of inclined cable in Fig.5 with $\sigma_{f}=.0035$ (from $\mathrm{HF}_{3}$ ).

11 Time response histories and Poincarè map showing the quasi-periodic response of inclined cable in Fig.5 with $\sigma_{f}=.006$ (from $\mathrm{HF}_{3}$ ).

12 PSDs of $q_{s}$-response histories corresponding to Figs.8d (a), 8e (b), 9 (c) and 11 (d).

13 Phase portraits showing the transition from periodically to chaotically-modulated dynamic response of inclined cable in Fig.7 with $\sigma=.08$.

14 The effect of initial conditions (set I $v s$ II) on dynamic responses in terms of phase portraits (a $v s$ b) and Poincarè maps ( $\mathrm{c} v s \mathrm{~d}$ ) of inclined cable in Fig.7 with $\sigma=.08$ and $F=.007$.

15 PSDs of $q_{s}$-response histories corresponding to Figs.14a, c and 14b, d.

16 A comparison of space-time varying coupled dynamic $(u, v)$ configurations and tension distributions $\left(T_{d} / T_{H}\right)$, based on fixed-point amplitudes with $F=.002, \sigma=.04$ and $\sigma_{f}=-.02$, over a half period of forced oscillation: corresponding to B1 (a-c) in Figs. $4 \mathrm{a}-4 \mathrm{~b}\left(\theta=30^{\circ}\right), \mathrm{B} 1$ (d-f) and B2 (g-i) in Figs.5a-5b $\left(\theta=45^{\circ}\right)$, respectively.

17 A comparison of space-time varying coupled dynamic $(u, v)$ configurations and tension distributions $\left(T_{d} / T_{H}\right)$, based on fixed-point (a-c) and time-varying (d-f) amplitudes with $\theta=45^{\circ}, F$ $=.002, \sigma=.04$ and $\sigma_{f}=.0225$, over a half period of forced oscillation: corresponding to B1 (a-c) in Figs.5a-5b and Fig.8e with $t \approx 211.2-216.2$ (d-f), respectively.

\section{List of Tables}

Table

1 A comparison of possible asymptotic solutions for different planar modal interaction cases.

2 Given parameters and properties for different avoidance inclined cables.

3 A comparison of effective interaction coefficients for different avoidance inclined cables. 
(a)
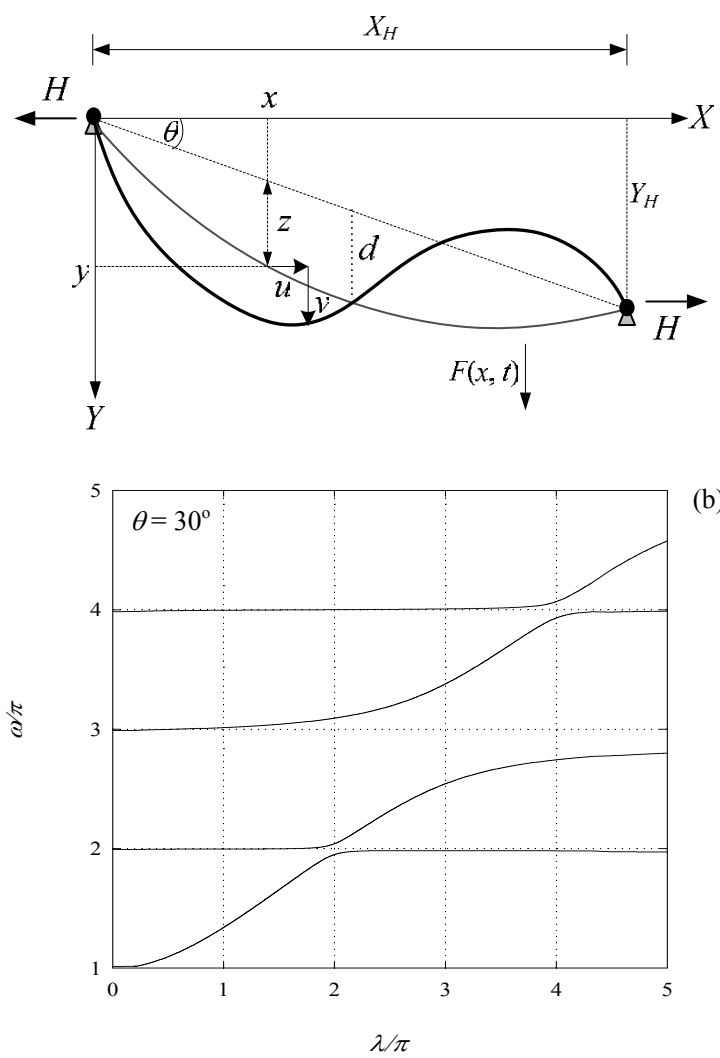

(b)

Figure 1 

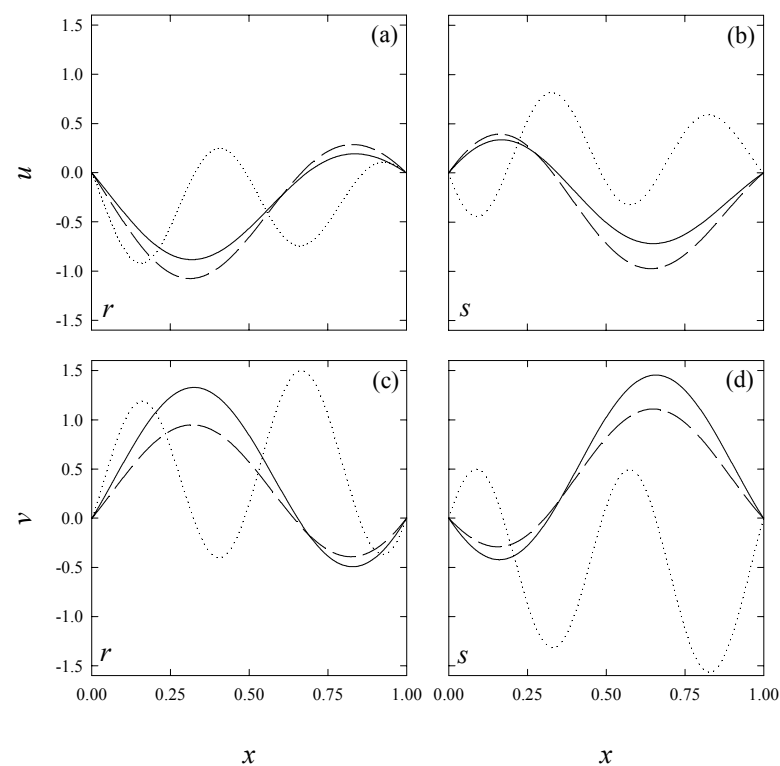

Figure 2 

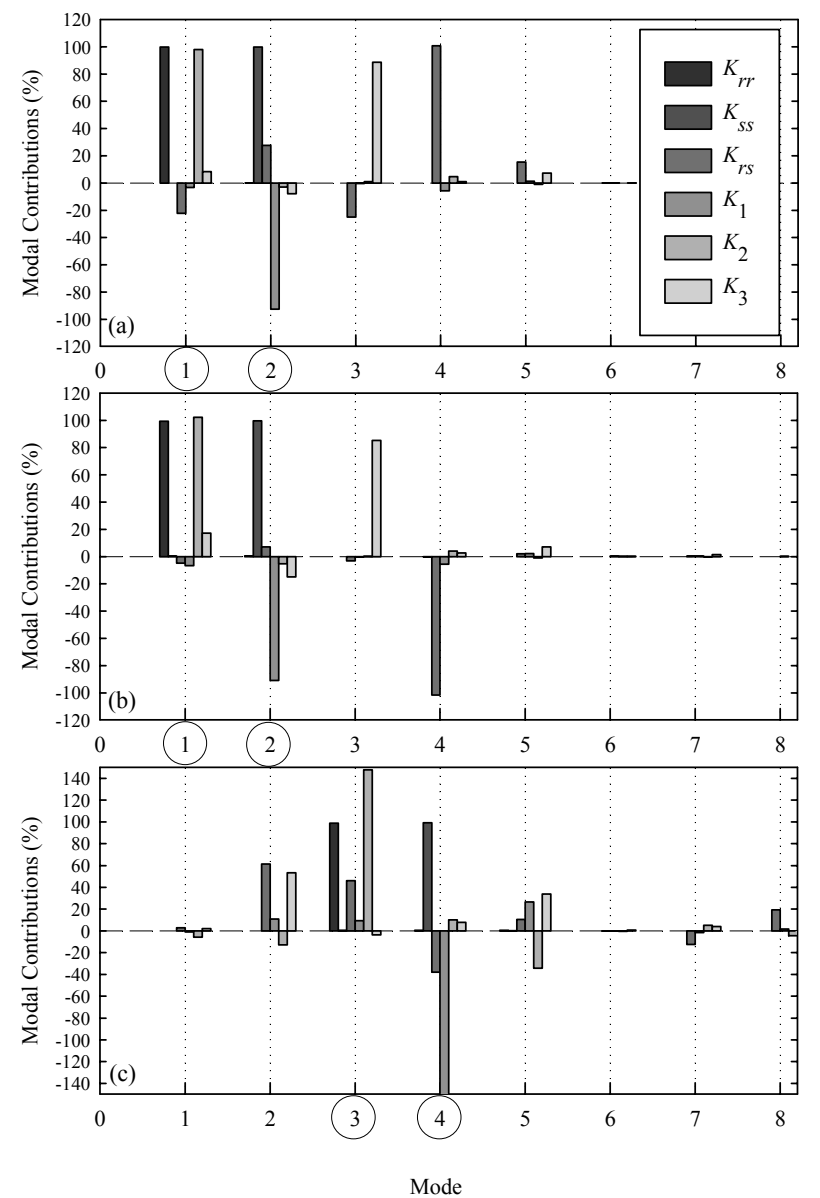

$\underline{\text { Figure } 3}$ 

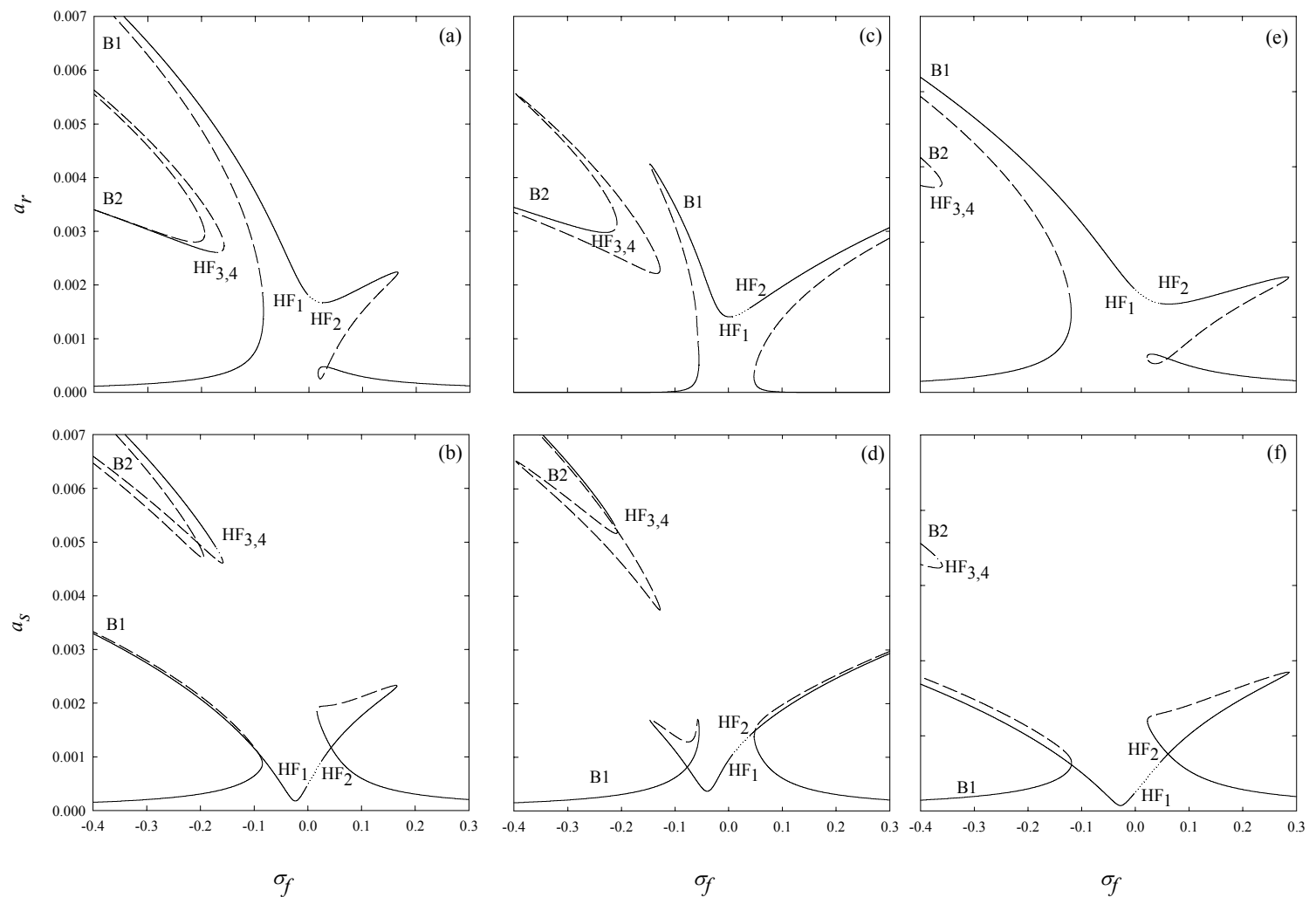

Figure 4 

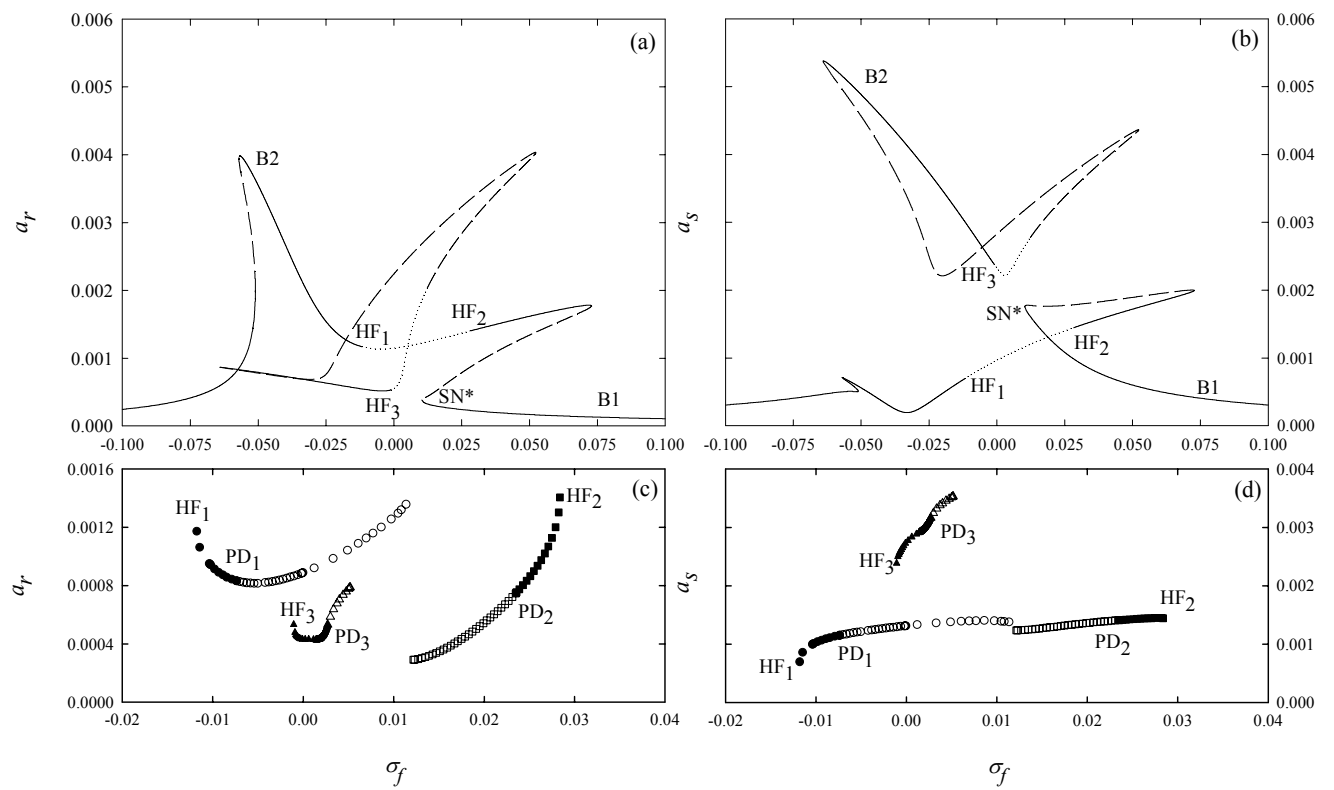

Figure 5 

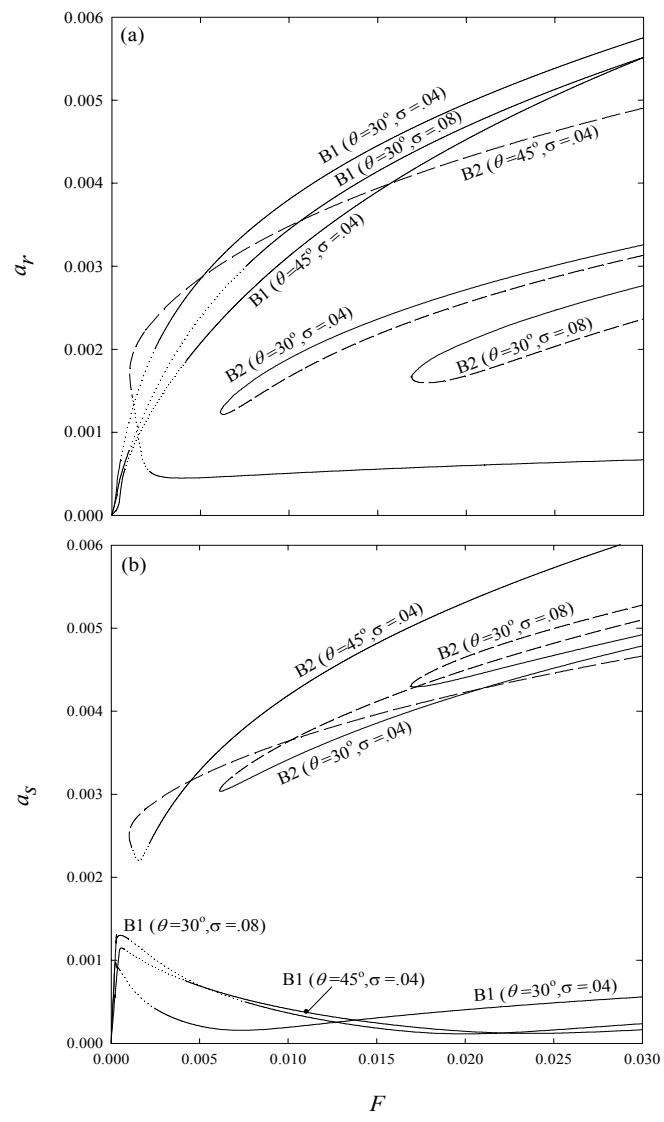

$\underline{\text { Figure } 6}$ 


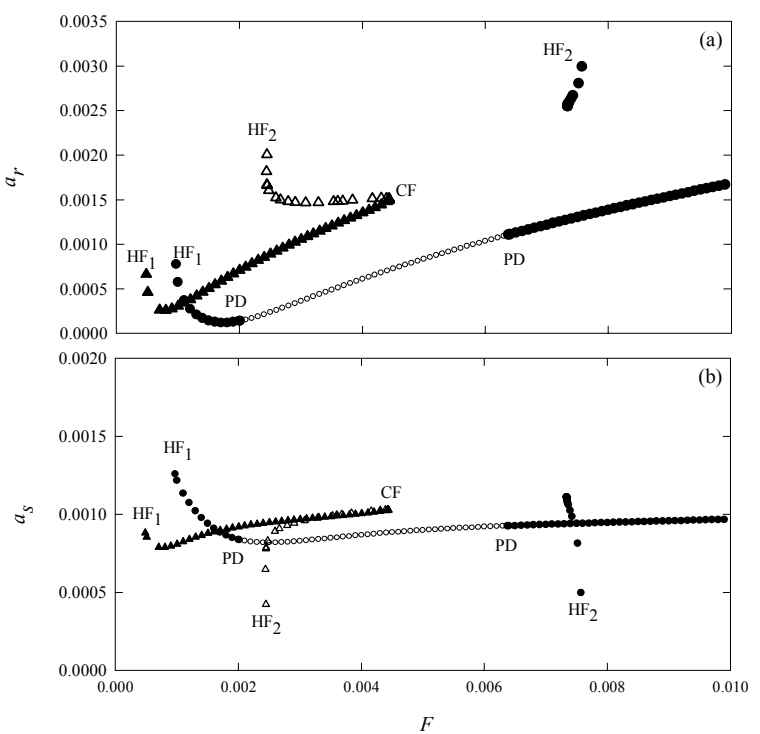

Figure 7 

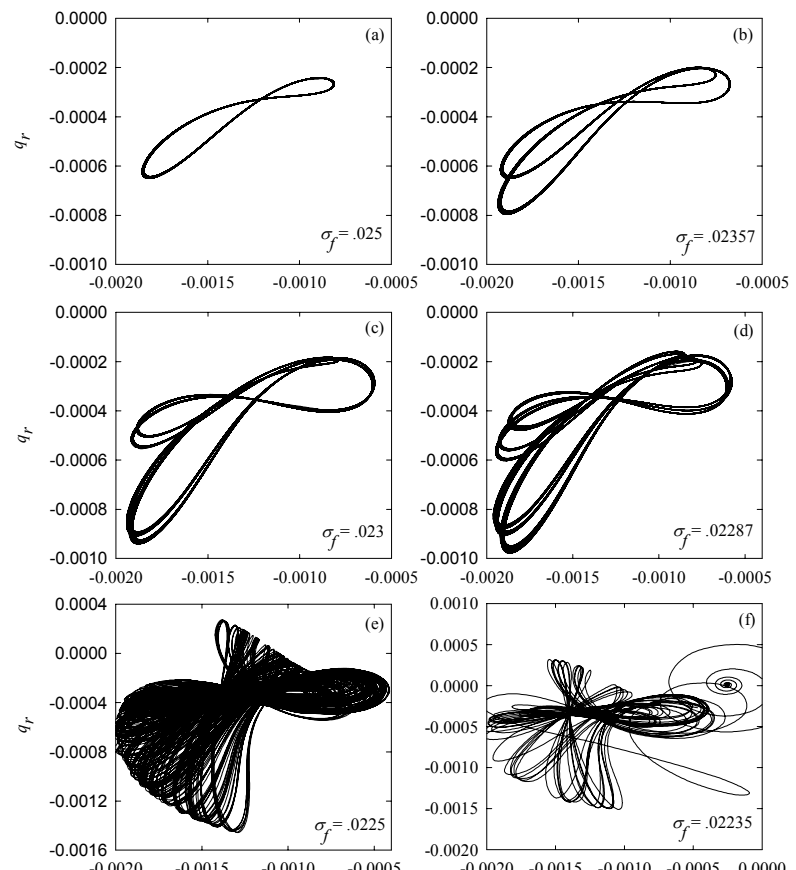

Figure 8
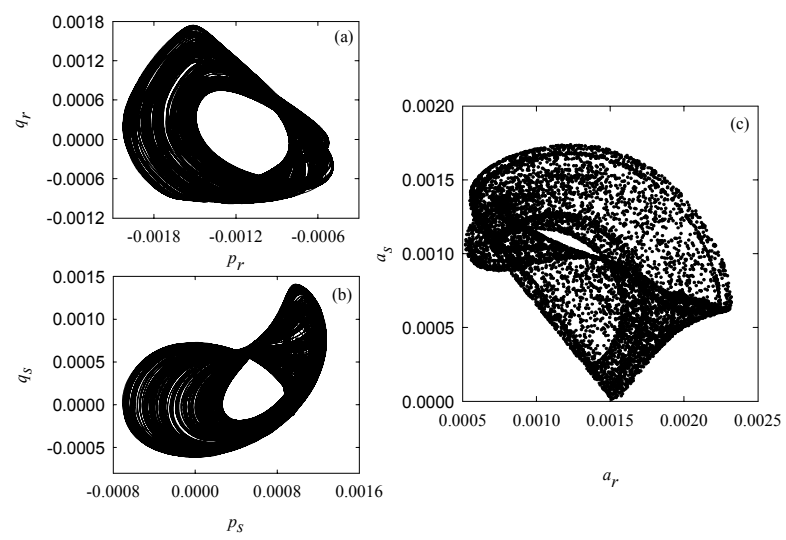

$\underline{\text { Figure } 9}$ 


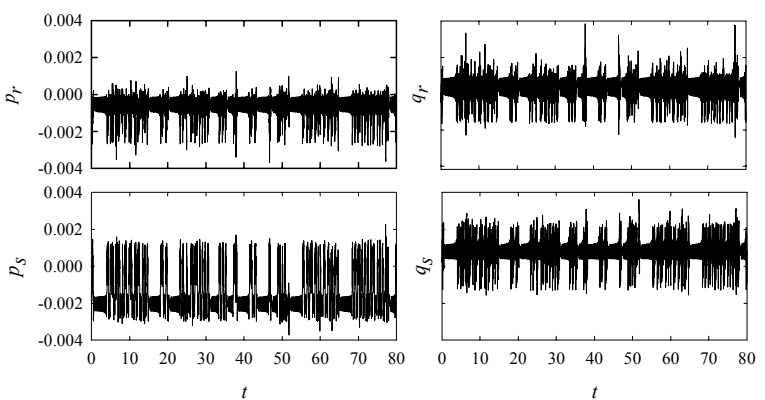

Figure 10

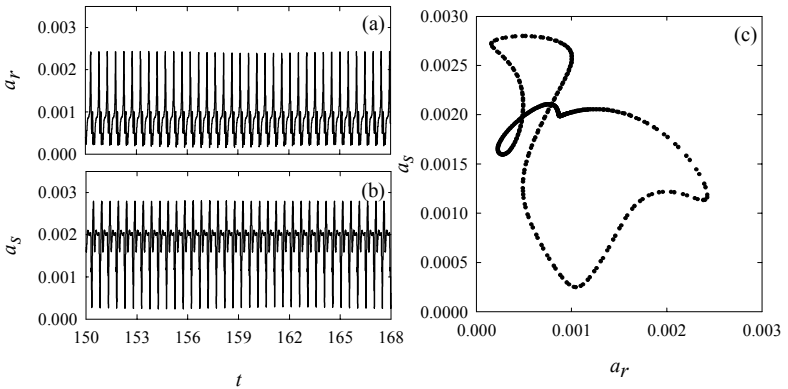

Figure 11 

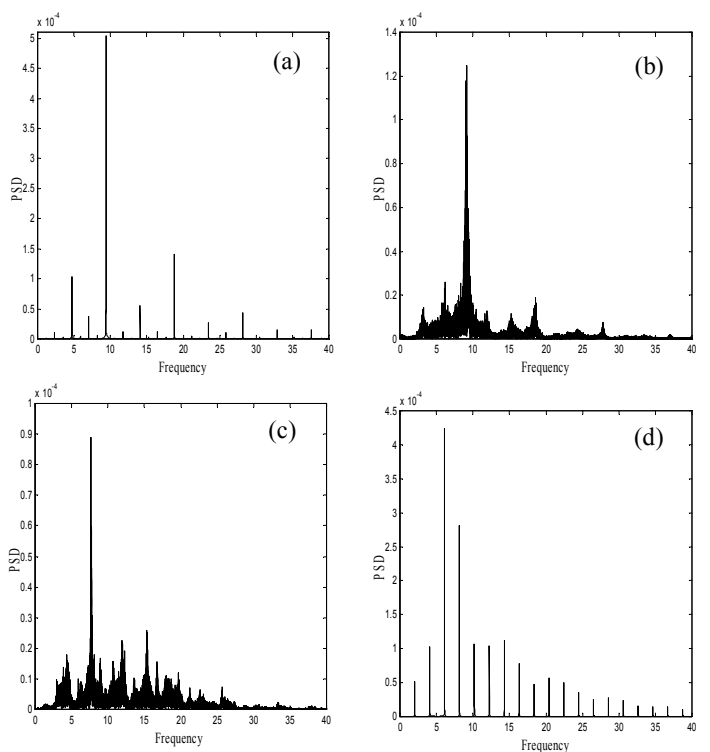

Figure 12 


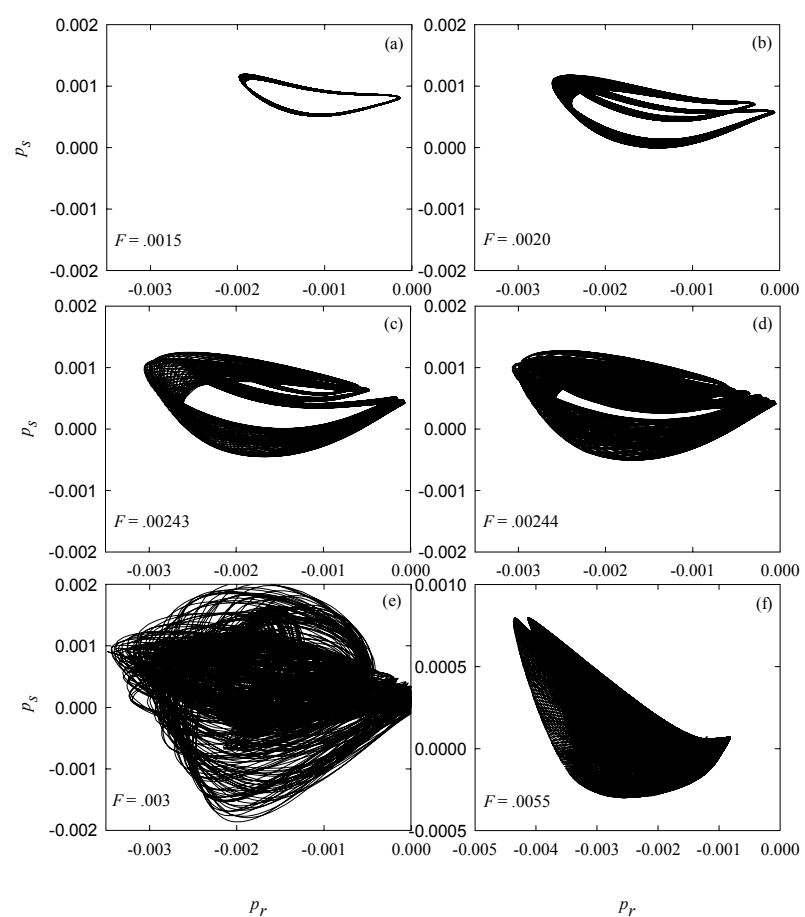

Figure 13 

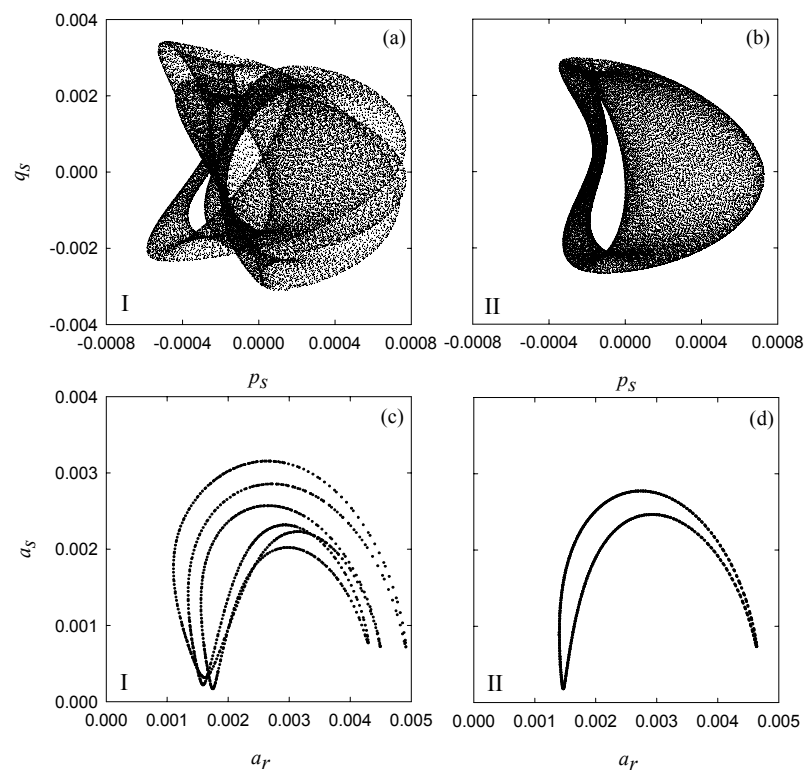

Figure 14 

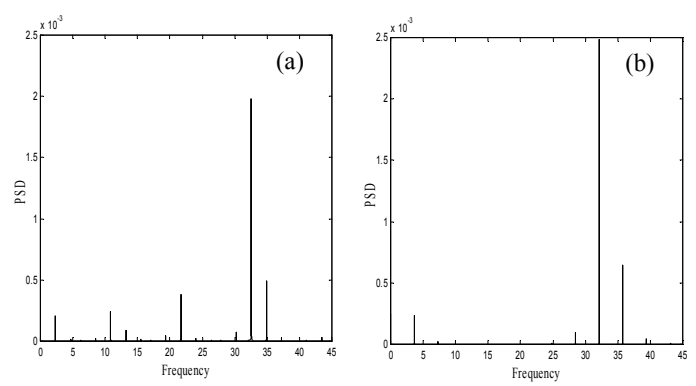

Figure 15 

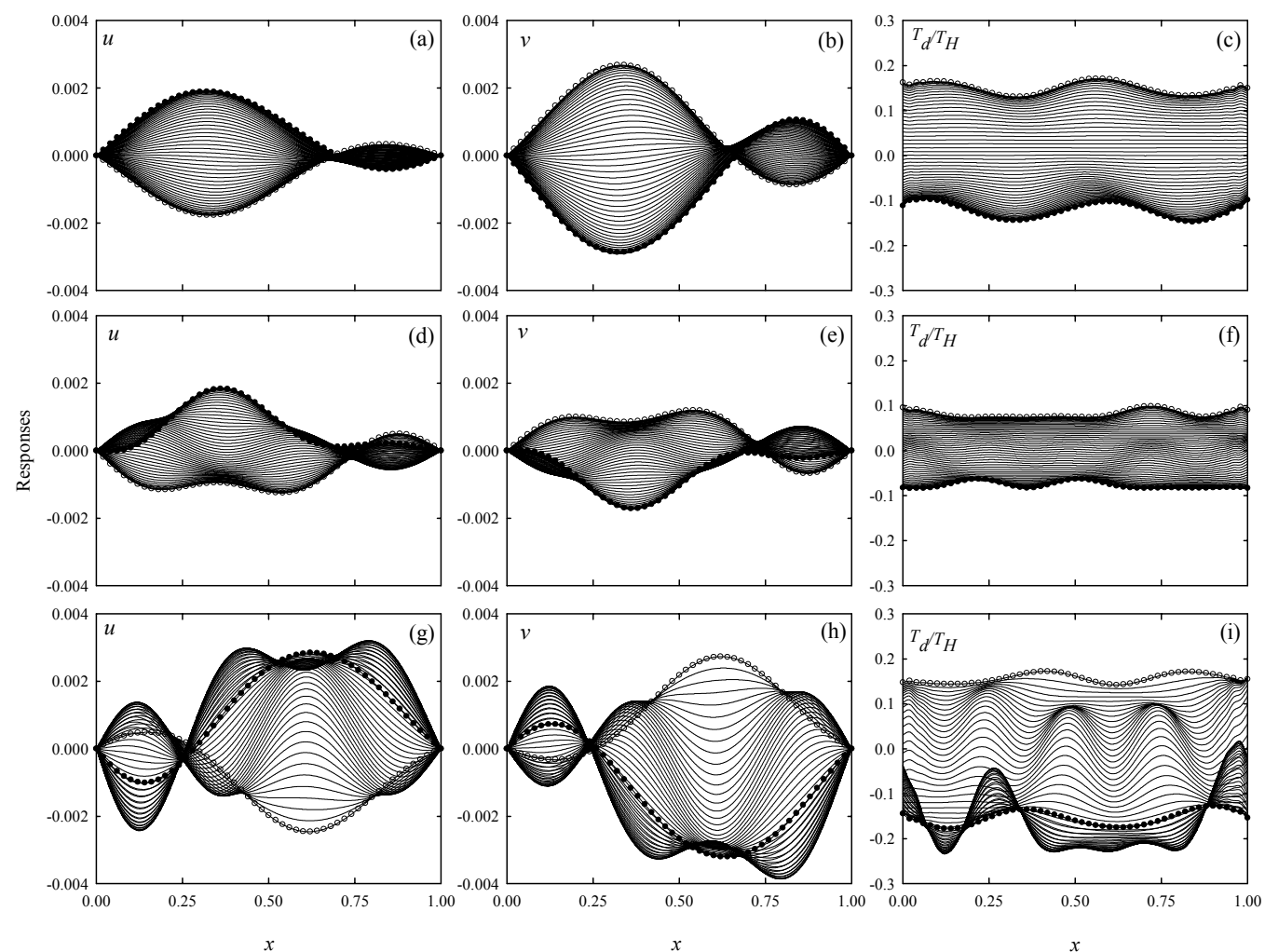

Figure 16 

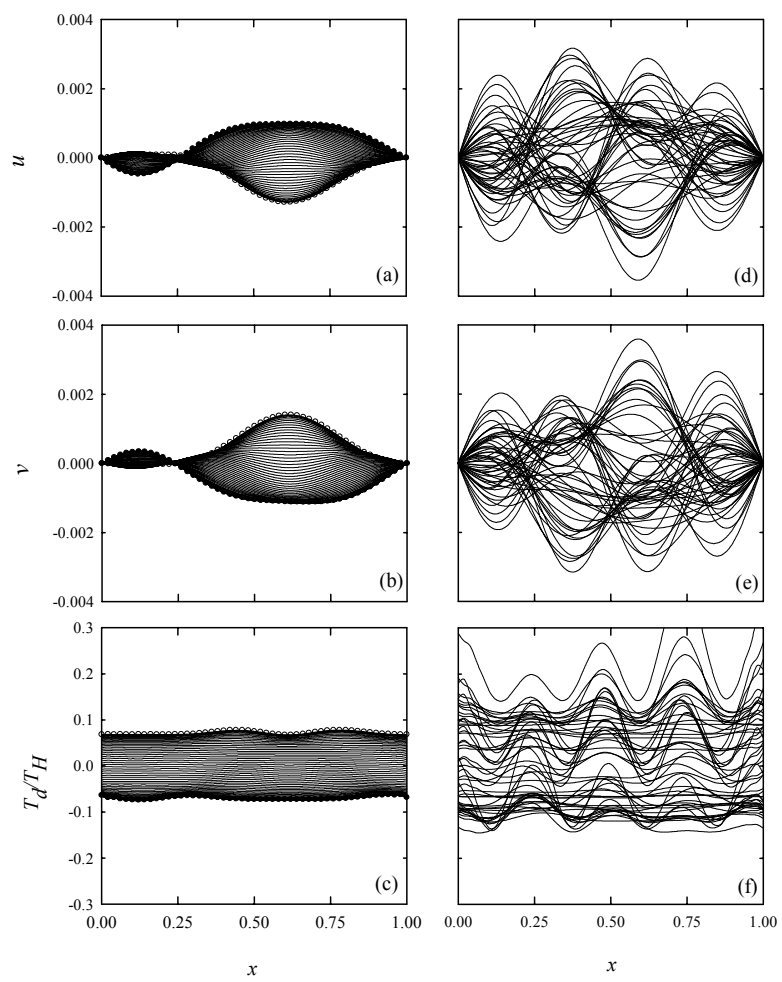

Figure 17 


\section{Table 1}

\begin{tabular}{ccccccc}
\hline \multirow{2}{*}{$s: r$} & \multirow{2}{*}{$n / \pi$} & $n$ & \multicolumn{2}{c}{ horizontal (sym.) cables } & \multicolumn{2}{c}{ inclined (asym.) cables } \\
\cline { 4 - 7 } & & $\Omega=\omega_{s}+\varepsilon^{n} \sigma_{f}$ & $\Omega=\omega_{r}+\varepsilon^{n} \sigma_{f}$ & $\Omega=\omega_{s}+\varepsilon^{n} \sigma_{f}$ & $\Omega=\omega_{r}+\varepsilon^{n} \sigma_{f}$ \\
\hline $1: 1$ & $\mathrm{CR}$ vs. AV & 2 & $\mathrm{UC} / \mathrm{C}$ & $\mathrm{UC} / \mathrm{C}$ & $\mathrm{C}$ & $\mathrm{C}$ \\
$2: 1$ & $\begin{array}{c}\text { CR vs. AV } \\
\text { Non-CR vs. Non-AV }\end{array}$ & 1 & $\mathrm{UC} / \mathrm{C}$ & $\mathrm{C}$ & $\mathrm{UC} / \mathrm{C}$ & $\mathrm{C}$ \\
& & & & & \\
$3: 1$ & Non-CR vs. Non-AV & 2 & $\mathrm{UC} / \mathrm{C}$ & $\mathrm{C}$ & $\mathrm{UC} / \mathrm{C}$ & $\mathrm{C}$ \\
\hline
\end{tabular}

UC (C): Uncoupled-mode (Coupled-mode) solution.

CR (AV): Crossover (Avoidance).

Table 2

\begin{tabular}{rrrrrrr}
\hline$\lambda / \pi$ & $\theta$ & \multicolumn{1}{c}{$\alpha$} & $d$ & $r, s$ & \multicolumn{1}{c}{$\omega_{r}$} & \multicolumn{1}{c}{$\omega_{s}$} \\
\hline 2 & $30^{\circ}$ & 738.802 & .041 & 1,2 & 5.694 & 5.958 \\
2 & $45^{\circ}$ & 907.707 & .062 & 1,2 & 5.072 & 5.489 \\
4 & $30^{\circ}$ & 1168.626 & .065 & 3,4 & 11.415 & 11.804 \\
\hline
\end{tabular}

Table 3

\begin{tabular}{|c|c|c|c|}
\hline \multirow{2}{*}{$K$} & \multicolumn{2}{|c|}{$\theta=30^{\circ}$} & $\theta=45^{\circ}$ \\
\hline & $\lambda / \pi \approx 2$ & $\lambda / \pi \approx 4$ & $\lambda / \pi \approx 2$ \\
\hline$K_{r r}$ & $18,431.010$ & $2,919,187.945$ & $-7,812.204$ \\
\hline$K_{s S}$ & $101,728.107$ & $-480,020.584$ & $63,702.183$ \\
\hline$K_{r s}$ & $-450,390.519$ & $-10,629,041.605$ & $-582,906.364$ \\
\hline$K_{1}$ & $254,518.048$ & $-7,991,366.439$ & $103,897.379$ \\
\hline$K_{2}$ & $289,082.594$ & $-5,376,948.755$ & $135,892.499$ \\
\hline$K_{3}$ & $-218,734.377$ & $-3,022,752.641$ & $-95,349.086$ \\
\hline
\end{tabular}

